\title{
Approach to chaos: Universal quantitative properties of one-dimensional maps
}

\author{
H. Kawai and S.-H. H. Tye \\ Floyd R. Newman Laboratory of Nuclear Studies, Cornell University, Ithaca, New York 14853
}

(Received 31 May 1984)

\begin{abstract}
Using the renormalization-group method, numerical results, and analytical arguments, we obtain the universal metric properties of one-dimensional iterated maps which exhibit period doublings. Such maps can be classified according to their behaviors around the extremum. For maps with the extremum (at $x=0$ ) of the symmetric form $f(x) \sim|x|{ }^{z} b(x)$, with $z>1$, where the function $b(x)$ modifies $|x|^{z}$ by less than any power of $|x|$, the quantitative universal properties of the perioddoubling approach to chaos is described for asymptotically large $n$, by $\delta_{n}=\left(\lambda_{n}-\lambda_{n-1}\right) /\left(\lambda_{n+1}-\lambda_{n}\right)$ $=\delta[1+\epsilon(n) \tau \ln \alpha]$ and $\alpha_{n}=-d_{n} / d_{n+1}=\alpha[1+\epsilon(n) \kappa \ln \alpha]$, where $\lambda_{n}$ is the value of the parameter at the $n$th period-doubling bifurcation, $d_{n}$ is the typical distance from the extremum at the $n$th bifurcation point, and $\epsilon(n)=[\partial \ln b(x) / \partial \ln |x|]_{|x| \sim \alpha^{-n}}$, where terms of order $n^{-2}$ are neglected. Here $\delta, \alpha, \kappa$, and $\tau$ are all functions of $z$ only; they obey the relations $d \ln \alpha / d z=\kappa \ln \alpha$ and $d \ln \delta / d z=\tau \ln \alpha$, where $\alpha$ and $\delta$ are the Feigenbaum constants. In particular, for $f(x) \approx|x|^{z}\left|\ln \left(x^{2}\right)\right|^{p} \quad|\ln | \ln \left(x^{2}\right)||^{q} \ldots$, where $z>1$, then $d_{n} \approx \alpha^{-n} n^{\kappa p}(\ln n)^{\kappa q} \ldots$ and $\lambda_{\infty}-\lambda_{n} \approx \delta^{-n} n^{\tau p}(\ln n)^{\tau q} \ldots$, where $\lambda_{\infty}$ is the critical value of the parameter beyond which is chaos. For $z=2, \alpha=2.50291, \delta=4.66920, \kappa=-0.44453$, and $\tau=0.36954$. For the sake of completeness, an analysis of maps with asymmetric extrema is also presented. In this case, $\alpha$ and $\delta$ are functions of $z$ as well as the size of the asymmetry.
\end{abstract}

\section{INTRODUCTION}

Because of their relative simplicity and experimental interest, one-dimensional (1D) mappings of the real axis

$$
f_{\lambda}: x \rightarrow x^{\prime}=f(\lambda, x)
$$

have been extensively studied. ${ }^{1-3}$ A number of results have been obtained for some classes of functions. In particular, Feigenbaum ${ }^{2}$ showed that if the function $f_{\lambda}$ exhibits period doublings as the parameter $\lambda$ is being increased, then $f_{\lambda}$ has an infinite sequence of perioddoubling bifurcations as $\lambda$ approaches the critical value $\lambda_{c}$ (beyond which $f_{\lambda}$ exhibits chaotic behavior). Furthermore, the behavior of the bifurcations is quantitatively universal and depends only on the shape of $f_{\lambda}$ near the extremum, $x=x_{c}$.

For example, if $\Delta f \approx\left|f_{\lambda}(x)-f_{\lambda}\left(x_{c}\right)\right| \approx\left|x-x_{c}\right|^{2}$, $z>1$, both the bifurcation speed $\delta$ and the reduction scale factor $\alpha$ depend only on the power $z$. We can always make the shift so that the extremum is at the origin, i.e., $x_{c}=0$. For polynomial maps in general, the extremum has the quadratic behavior, i.e., $z=2$; the corresponding values for $\delta$ and $\alpha$ are $\delta=4.6692016$ and $\alpha=2.5029079$. It has been shown that the same universal properties are present in higher-dimensional dissipative maps as well as in experiments.

Feigenbaum's analysis is applicable to $\Delta f \approx|x|^{z}$ for all finite $z, z>1$. In this paper we extend his analysis to the very general case where

$$
\Delta f \approx|x|^{z}\left|\ln \left(x^{2}\right)\right|^{p}|\ln | \ln \left(x^{2}\right)||^{q} \cdots,
$$

where $p, q, \ldots$ are real numbers. One-dimensional maps that exhibit infinite period-doubling bifurcation in general have their extrema of the form (1.1). [This form gives classification of 1D symmetric maps that exhibit repeated period doublings; actually there is another class of $1 \mathrm{D}$ asymmetric maps, ${ }^{4}$ see (1.13). The analysis of the latter case is straightforward and a brief discussion is included in Sec. V.] Iterated maps with the property (1.1) reveal additional universal properties. For $n$ asymptotically large, the value $\lambda_{n}$ of the parameter $\lambda$ at which $n$th bifurcation occurs behaves as

$$
\lambda_{\infty}-\lambda_{n} \approx \delta^{-n} n^{\tau p}(\ln n)^{\tau q} \ldots,
$$

while the typical distance away from the extremum at the $n$th bifurcation point is

$$
d_{n} \approx \alpha^{-n} n^{\kappa p}(\ln n)^{\kappa q} \ldots,
$$

where the function $f_{\lambda}$ has a stable orbit with period $2^{n}$ when $\lambda$ has a value $\lambda_{n}<\lambda \leq \lambda_{n+1} ; \kappa$ and $\tau$ are constants independent of $p, q, \ldots$ (In the special case where $p=q=\cdots=0$, Feigenbaum's result is recovered.) Like $\alpha$ and $\delta$, they are functions of $z$ only and they obey the following relations:

$$
\begin{aligned}
& \frac{d \ln \alpha}{d z}=\kappa \ln \alpha, \\
& \frac{d \ln \delta}{d z}=\tau \ln \alpha .
\end{aligned}
$$

Using the renormalization-group method developed by Wilson and others, ${ }^{5}$ we can analytically derive the results (1.2) and (1.3) for the cases of $p=$ positive integer and $q=0$. This analysis involves many interesting features. (For example, there appear $p+1$ marginal operators that take a Jordan's canonical form of order $p+1$.) This also enables us to analytically derive (1.4) and (1.5). $\kappa$ and $\tau$ can now be obtained via essentially three ways: 
(1) numerical data from any function $f_{\lambda}$ that exhibit (1.1),

(2) renormalization-group analysis, and

(3) Eqs. (1.4) and (1.5).

We obtain for $z=2$

$$
\begin{aligned}
& \kappa=-0.44453 \cdots, \\
& \tau=0.36954 \cdots .
\end{aligned}
$$

Although it is difficult to extend the renormalizationgroup method to $p \neq$ positive integer, the numerical data for $p \neq$ positive integer cases clearly indicate that the formulas (1.2) and (1.3) hold for any real number $p$, while $q=0$.

Assuming (1.2) and (1.3) to be correct for any real number $p$, an analytic-consistency argument shows that (1.2) and (1.3) are correct for large-integer values of $q$. Numerical data indicate that (1.2) and (1.3) are true also when $q$ is a small integer, $q=1,2, \ldots$, as well as when $q$ is not an integer. For $q \neq 0$ the approach to the asymptotic form (1.2) and (1.3) is extremely slow [corrections are of the order $\left.(\ln n)^{-1}\right]$. However, our argument does indicate that the universal constants $\alpha, \delta, \tau$, and $\kappa$ essentially determine the infinite cascade of period doubling. From the details of the analysis and the numerical data, we conjecture that (1.3) is true for all real numbers $p, q, \ldots$.

The renormalization-group results allow us to study the general symmetric case. In general, let us consider a function whose extremum at $x=0$ has the symmetric form

$$
f \approx|x|^{z} b(x), z>1,
$$

where the function $b(x)$ modifies the form $|x|^{z}$ by less than a power, i.e., as $x \rightarrow 0$,

$$
\begin{aligned}
& |x|^{v} b(x) \rightarrow 0, \\
& |x|^{-v} b(x) \rightarrow \infty,
\end{aligned}
$$

for any infinitesimal positive constant $v$. Then, we argue, for asymptotically large $n$, that

$$
\begin{aligned}
& \alpha_{n}=\frac{-d_{n}}{d_{n+1}}=\alpha[1+\epsilon(n) \kappa \ln \alpha], \\
& \delta_{n}=\frac{\lambda_{n}-\lambda_{n-1}}{\lambda_{n+1}-\lambda_{n}}=\delta[1+\epsilon(n) \tau \ln \alpha],
\end{aligned}
$$

where

$$
\epsilon(n)=\left.\frac{\partial \ln b(x)}{\partial \ln |x|}\right|_{|x| \approx \alpha^{-n}}
$$

and terms of order $n^{-2}$ are neglected. Readers who are only interested in this result can find the derivation in Sec. VB. The simple, intuitively clear argument used in the derivation in Sec. VB is justified by the careful, detailed renormalization-group analysis in Secs. III and IV. On the other hand, the derivation of (1.10)-(1.12) extends the renormalization-group results for $p=$ integer in (1.1)(1.3) to all real values of $p, q, \ldots$.

The constants $\alpha, \delta, \kappa$, and $\tau$ also determine the asymptotic band-bifurcation properties on the chaotic regime. ${ }^{3}$ It will be interesting to study the effect of noise on such systems.

For the sake of completeness, we also discuss the case $e^{4}$ where the extremum is asymmetric, i.e.,

$$
\Delta f \approx \begin{cases}a(1+\epsilon)|x|^{z} \text { for } x>0 \\ a(1-\epsilon)|x|^{z} \text { for } x<0,\end{cases}
$$

where $\epsilon \neq 0$. In this case the universal equation for the universal function $g(x)$ is different from Feigenbaum's case, so that $\alpha$ and $\delta$ are functions of both $z$ and the magnitude of the asymmetry $\epsilon$.

Feigenbaum's analysis ${ }^{2}$ is applicable to all functions with the extrema of the form $|x|^{z}$. As is clear from general arguments, $\alpha$ and $\delta$ have the following behaviors: as $z \rightarrow 1, \alpha \rightarrow \infty$ and $\delta \rightarrow 2$; as $z \rightarrow \infty, \alpha \rightarrow 1$ and $\delta \rightarrow \infty$. Since the period-doubling approach to chaos requires finite, well-defined $\alpha$ and $\delta$, the extremum of the function can only be modified by behaviors slower than powers of $x$, if the period-doubling property is to be preserved. We argue that the only generalization of the Feigenbaum case is the introduction of logarithmic and/or asymmetric behaviors. This leads us to believe that our analysis is a complete analysis of the one-dimensional maps that exhibit the period-doubling approach to chaos. Note that all the functions that are considered in our analysis are at least once differentiable at the extrema.

The analysis of the universal properties of 1D maps certainly gives us a better understanding of the mathematical structures of iterated maps. It also demonstrates how the renormalization-group method ${ }^{5}$ can be used both as an analytical and a numerical tool. Although we do not know of any physical application of the type of maps discussed here, it is possible that they are relevant in certain situations. In quantum-field theory a massless scalar field $x$ (e.g., a field responsible for spontaneous symmetry breaking) has a unique renormalizable self-interaction $f(x) \approx \lambda x^{4}$. Quantum effects due to its interactions with gauge fields and/or massless fermion fields and/or massless scalar fields will change it to $f(x) \approx x^{4} \ln \left(x^{2}\right)$, which is mainly due to the presence of infrared singularities. ${ }^{6}$ Such a functional form plays a very important role in the study of the early universe in cosmology.

On the other hand, effective one-dimensional maps of systems with finite degrees of freedom sometimes show nonpolynomial extrema. For dissipative systems, one can always obtain one-dimensional maps via Poincaré section or via projection. Even if the original system has only polynomial-type couplings, nonpolynomial extremum can appear in the effective one-dimensional map obtained by these procedures. For example, in the Lorentz model ${ }^{7}$ there is a variable $Z$ which measures the distortion of the vertical temperature profile; the one-dimensional map of $M_{n}$, the $n$th maximum value of $Z$, versus $M_{n+1}$, the value of the following maximum, displays a nonpolynomial extremum. One can easily envision the possibility of extrema with logarithmic modifications to the polynomial form in some nontrivial model.

If a logarithmic modification such as (1.1) is present, typically the transient behavior can be more persistent, and the values $\delta_{n}$ and $\alpha_{n}$ approach their respective universal values $\delta$ and $\alpha$ at a much slower rate. A comparison 
TABLE I. Values of the superstable points $\tilde{\lambda}_{n}$ for the logistic equation (2.1) and the corresponding $\delta_{n}$ and $\alpha_{n}$ for the $2^{n}$ th iteration. Our calculations of $\tilde{\lambda}_{n}$ are performed with a $\sim 30$ significant-figure accuracy. Here, we display only the first 20 significant figures.

\begin{tabular}{rccc}
\hline \hline$n$ & $\tilde{\lambda}_{n}$ & $\delta_{n}$ & $\alpha_{n}$ \\
\hline 1 & 0.80901699437494742410 & & \\
2 & 0.87464042483192538000 & 4.70894301354050 & 2.62736996425867 \\
3 & 0.88866021569220621634 & 4.68077099801070 & 2.53998436842616 \\
4 & 0.89166684496406712849 & 4.66295961111410 & 2.50538499147915 \\
5 & 0.89231088290927758445 & 4.66840392591840 & 2.50382008126151 \\
6 & 0.89244882343748615513 & 4.66895374096762 & 2.50305093206343 \\
7 & 0.89247836635558712871 & 4.66915718132884 & 2.50294452010044 \\
8 & 0.89248469355832637195 & 4.66919100248510 & 2.50291497019195 \\
9 & 0.89248604865201623331 & 4.66919947054773 & 2.50290948675676 \\
10 & 0.89248633887161714522 & 4.66920113460104 & 2.50290820885741 \\
11 & 0.89248640102776960953 & 4.66920150951355 & 2.50290794798545 \\
12 & 0.89248641433971412493 & 4.66920158752239 & 2.50290789053247 \\
13 & 0.89248641719072499209 & 4.66920160451219 & 2.50290787842346 \\
14 & 0.89248641780132421363 & 4.66920160811594 & 2.50290787580589 \\
15 & 0.89248641793209586855 & 4.66920160889207 & 2.50290787524828 \\
\hline \hline
\end{tabular}

of Tables I and II should illustrate this point. This means an accurate experimental measurement may reveal the presence or absence of logarithmic factors. From Table I we see, for $n \geq 4$, that the values of $\delta_{n-1}$ and $\alpha_{n-1}$ are essentially those of $\delta$ and $\alpha$ (difference $<0.1 \%$ ) while the values of $\delta_{n-1}(p=1)$ and $\alpha_{n-1}(p=1)$ in Table II deviate from the asymptotic values $\delta$ and $\alpha$ by a few percent. In experiments that produce power spectra the drop in power per period doubling will deviate from the value predicted. ${ }^{8}$ In fact, an accurate experiment that can reach the fifth or sixth period doubling may even provide a measurement of $p, \kappa$, and $\tau$.

The renormalization-group (RG) method is a mathematical technique to treat problems which have scaling properties. It plays a crucial role in many areas of physics, e.g., quantum-field theory and critical phenomena. However, in these applications of the RG method, an in-depth knowledge of the relevant physics is necessary.
This prevents many nonphysicists from learning the RG method, which is simply a powerful mathematical tool. In this work the RG method plays a central role in the analysis. Readers with no physics background can appreciate the power and the working of the renormalizationgroup method itself.

This paper is organized as follows. In Sec. II the numerical data for $p \neq 0$ are presented. The data clearly suggest the existence of the universal constants $\kappa$ and $\tau$. In Sec. III a renormalization-group approach is presented for the case where $p=1, q=0$. This allows us to calculate $\kappa$ and $\tau$ from the renormalization-group equations. Because of the length of this section, many of the details are relegated to the three appendixes. The generalization to $p=$ positive integer is carried out in Sec. IV. This allows us to derive analytically the relations (1.4) and (1.5). In Sec. IV we also discuss the numerical results from the renormalization-group analysis. In Sec. $\mathrm{V}$ we discuss the

TABLE II. Values of the superstable points $\tilde{\lambda}_{n}$ for Eq. (2.14) and the corresponding $\delta_{n}$ and $\alpha_{n}$.

\begin{tabular}{cccc}
\hline \hline$n$ & $\tilde{\lambda}_{n}$ & $\delta_{n}$ & $\alpha_{n}$ \\
\hline 1 & 0.50865430728735759782 & & \\
2 & 0.51191485766282221896 & 2.65424737874948 & 1.85527781802036 \\
3 & 0.51275103541248054278 & 3.89935079807725 & 2.44546591647508 \\
4 & 0.51294756273179610575 & 4.25476596623026 & 2.56495229940641 \\
5 & 0.51299243228181407397 & 4.37997080953258 & 2.59157731684006 \\
6 & 0.51300255071123946488 & 4.43443820494254 & 2.59267005019762 \\
7 & 0.51300481608702165221 & 4.46655672094324 & 2.58833931039727 \\
8 & 0.51300532069583075942 & 4.48937026326478 & 2.58313969396407 \\
9 & 0.51300543265387024716 & 4.50712437816892 & 2.57816402368166 \\
10 & 0.51300545741485009012 & 4.52155125515176 & 2.57362847389220 \\
11 & 0.51300546287654943609 & 4.53356698611310 & 2.56954160520184 \\
12 & 0.51300546407857487854 & 4.54374687348686 & 2.56586359439183 \\
13 & 0.51300546434261193400 & 4.55248768153859 & 2.56254693660170 \\
14 & 0.51300546440051381927 & 4.56007700305858 & 2.55954683006910 \\
15 & 0.51300546441319288897 & 4.56672978869750 & 2.55682361137937 \\
16 & 0.51300546441596571867 & 4.57261030547918 & 2.55434293552868 \\
\hline \hline
\end{tabular}


generalization to the case (1.1). The evidence for the form (1.2) and (1.3) is partly numerical and partly analytic. Our argument clearly indicates the behaviors of the most general symmetric case (1.8)-(1.12). In this section the theory of the asymmetric case (1.13) is also briefly discussed. Section VI contains the conclusion as well as some remarks. Appendix A discusses the effect of a change in the choice of the marginal operator $s(x)$. Appendix $B$ contains the proof that $\kappa$ and $\tau$ are invariant under a change of the operator $s(x)$. A number of relations among the coefficients of the set of renormalization-group equations are derived. Appendix $\mathbf{C}$ gives some properties of $s(x)$ in relation to $\kappa$. Here, the functional relation (1.4) is derived using a different approach than that in Sec. IV.

\section{NUMERICAL DATA AND INTERPRETATION}

To be specific, let us consider the following onedimensional iterative map (the logistic equation)

$$
x_{n+1}=f\left(\lambda, x_{n}\right)=4 \lambda x_{n}\left(1-x_{n}\right),
$$

where $x_{n}$ is a real number, $x_{n} \in[0,1]$. This map is well studied. ${ }^{1,9}$ Our review allows us to introduce the notations and set up the stage for generalization. Depending on the value of $\lambda$ this map may or may not have a stable orbit with a definite period. It is easy to show that if $\frac{1}{4}<\lambda \leq \frac{3}{4}$ there is a stable (i.e., attractive) fixed point at $x^{*}=1-1 / 4 \lambda$, i.e.,

$$
x^{*}=f\left(\lambda, x^{*}\right) \text {, }
$$

such that repeated iterations of (2.1) will bring $x_{n}$ towards $x^{*}$. This is a stable fixed point. As $\lambda$ increases above $\frac{3}{4}$ this fixed point becomes unstable while a pair of new stable points, $x_{1}$ and $x_{1}^{\prime}$, appears so that

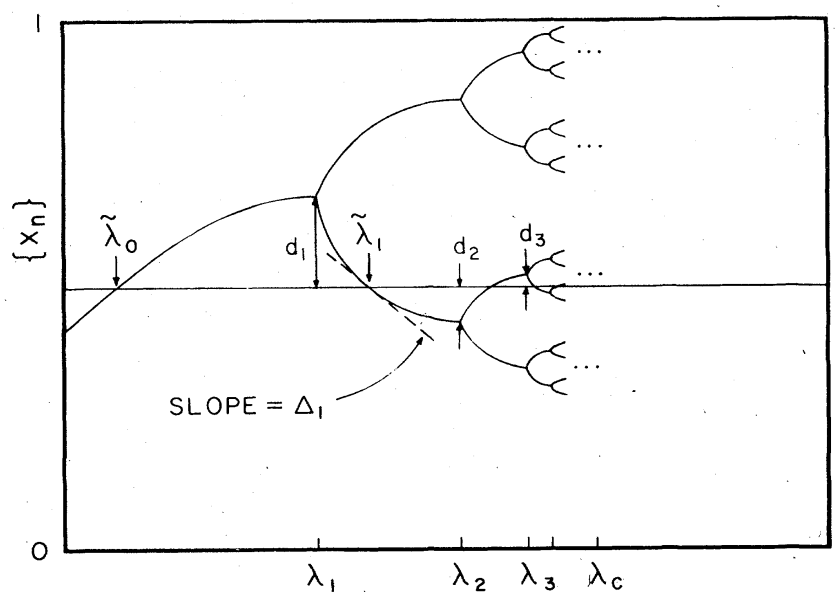

FIG. 1. Attractor, i.e., the stable orbit with period $2^{n}$, vs the bifurcation parameter $\lambda$ for a typical map that exhibits a cascade of period doublings. The figure is schematic. $\tilde{\lambda}_{i}$ are the superstable points. $\Delta_{i}$ is the slope at the point $\tilde{\lambda}_{i}$. The extremum is taken to be at $x_{c}=\frac{1}{2} . \lambda_{c}$ is the critical point.

$$
\begin{aligned}
x_{1}=f\left(\lambda, x_{1}^{\prime}\right) & \\
x_{1}^{\prime}=f\left(\lambda, x_{1}\right)=f\left(\lambda, f\left(\lambda, x_{1}^{\prime}\right)\right) & =f \odot f\left(\lambda, x_{1}^{\prime}\right) \\
& =f^{2}\left(\lambda, x_{1}^{\prime}\right) .
\end{aligned}
$$

They are stable in the sense that points in their neighborhood will approach them under repeated iterations. They form a stable orbit with period 2. $\lambda_{1}$ is the value of $\lambda$ at which the bifurcation from period 1 to period 2 occurs. In general $\lambda_{n}$ is the value of $\lambda$ at which the $n$th bifurcation occurs.

In Fig. 1 the stable orbit with period $2^{n},\left\{x_{n}\right\}=\left\{x_{n}^{(i)}\right.$, $\left.i=1,2, \ldots, 2^{n}\right\}$, is shown as a function of $\lambda$. This figure is schematic and is generic for all 1D iterated maps which exhibit cascade of period-doubling bifurcation and have $x_{c}=\frac{1}{2}$. Let us define

$$
\begin{aligned}
& \delta_{n}=\frac{\lambda_{n}-\lambda_{n-1}}{\lambda_{n+1}-\lambda_{n}}, \\
& \alpha_{n}=-\frac{d_{n}}{d_{n+1}}
\end{aligned}
$$

then for large $n, \delta_{n} \rightarrow \delta$ and $\alpha_{n} \rightarrow \alpha$, where $\alpha$ and $\delta$ are the Feigenbaum constants. It is clear that for some value of $\lambda\left(\equiv \widetilde{\lambda}_{n}\right), x_{c}$ is just on the stable orbit of period $2^{n}$, where

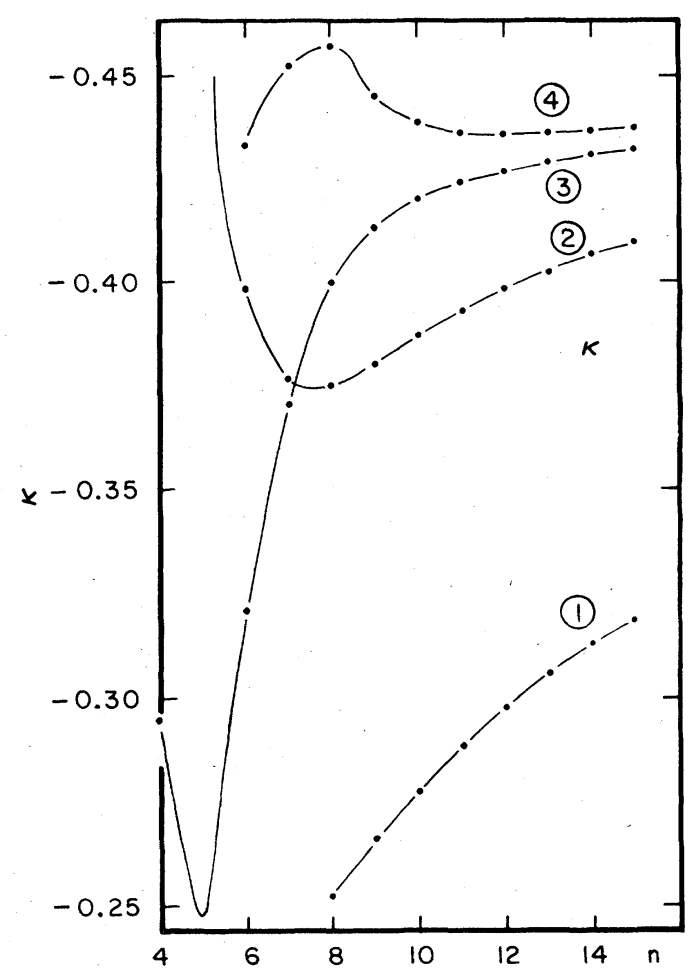

FIG. 2. Values of $\kappa$ from Table III, which are extracted from the numerical data of $\alpha_{n}$ in Table II for the function (2.16). Curve 1 is the one-parameter fit, while curve 2 includes a correction term of the form $A / n$. Curve 3 includes correction terms of the form $(A / n)+\left(B / n^{2}\right)$ and curve 4 includes terms of the form $(A / n)+\left(B / n^{2}\right)+\left(C / n^{3}\right)$. Curves $1,2,3$, and 4 correspond to $\kappa_{1}, \kappa_{2}, \kappa_{3}$, and $\kappa_{4}$ in Table III. The inputs are discrete points at integer values of $n$; the curve is to guide the reader. 
$\lambda_{n}<\tilde{\lambda}_{n}<\lambda_{n+1}$. For large enough $n$, we can use $\tilde{\lambda}_{n}$ for an alternative, equivalent (but not equal) definition for $\delta_{n}$,

$$
\delta_{n}=\frac{\tilde{\lambda}_{n}-\tilde{\lambda}_{n-1}}{\tilde{\lambda}_{n+1}-\tilde{\lambda}_{n}} .
$$

$\tilde{\lambda}_{n}$ are known as the superstable points. We note that the distance $d_{n}$ vanishes at the superstable points. So, instead of Eq. (2.5), an equivalent scale factor can be defined at $\tilde{\lambda}_{n}$,

$$
\alpha_{n}=-\delta_{n} \frac{\Delta_{n}}{\Delta_{n+1}},
$$

where $\Delta_{n}$ is the slope at $\tilde{\lambda}_{n}$. In Table I the $\tilde{\lambda}_{n}, \delta_{n}$, and $\alpha_{n}$ are shown for $n$ up to $n=15$ for the logistic equation (2.1). It is clear that $\alpha_{n}$ and $\delta_{n}$ rapidly approach their respective universal values $\alpha$ and $\delta$. This implies

$$
\begin{aligned}
& \Delta \tilde{\lambda}_{n} \approx \tilde{\lambda}_{n}-\tilde{\lambda}_{n-1} \approx \delta^{-n}, \\
& d_{n} \approx \alpha^{-n} .
\end{aligned}
$$

This universal quantitative behavior is present because the behavior of the iterated map for large $n$ is dominated by its property around the extremum. Hence, if $f_{\lambda}(x)$ is a general polynomial, its extremum will be quadratic (i.e., $z=2$ ). Therefore its period-doubling behavior will be given by (2.8) and (2.9) for sufficiently large $n$. In practice (2.8) and (2.9) are good approximations when $n \geq 3$ or 4. If we change (2.1) to

$$
x_{n+1}=\lambda\left(1-c\left|x_{n}-\frac{1}{2}\right|^{z}\right), \quad z>1
$$

then the period-doubling-bifurcation behavior will again obey (2.8) and (2.9). But the values of $\alpha$ and $\delta$ will be different, i.e., $\alpha=\alpha(z)$ and $\delta=\delta(z)$. For $z \rightarrow 1, \alpha \rightarrow \infty$ and $\delta \rightarrow 2$. For $z \rightarrow \infty, \alpha \rightarrow 1$ and $\delta \rightarrow \infty$. The period-doubling property is present for an appropriate range of values of the coefficient $c$. It is easy to convince oneself (via numerical data, for example) that $\alpha(z)$ and $\delta(z)$ are differentiable functions of $z, z \in(1, \infty)$.

Now let us consider the following map, $z>1$ :

$$
x_{n+1}=\lambda\left(1-c\left|x_{n}-\frac{1}{2}\right|^{z}\left|\ln \left(x_{n}-\frac{1}{2}\right)^{2}\right|^{p}\right),
$$

where $p$ is a real number. Here, the extremum at $x_{c}=\frac{1}{2}$ is modified by logarithm. The effect of the logarithmic modification is less than that of a power modification, no matter how small the change in power is. Therefore, we expect $\alpha_{n}, \delta_{n} \rightarrow \alpha(z)$ and $\delta(z)$. However, the speed of approach may be modified. For $p \neq 0$ we expect $\Delta \lambda_{n}$ in Eq. (2.8) and $d_{n}$ in Eq. (2.9) to be functions of $p$ as well as $\alpha$, $\delta$, and $n$. As we shall see, the numerical data indicate that, for any $p$ and $z$, we have, for large $n$,

$$
\begin{aligned}
& \tilde{\lambda}_{\infty}-\tilde{\lambda}_{n} \approx \delta^{-n} n^{p \tau}, \\
& d_{n} \approx \alpha^{-n_{n} n^{p \kappa},}
\end{aligned}
$$

where $\alpha, \delta, \kappa$, and $\tau$ are functions of only $z$.

In Table II the numerical data for $z=2, p=1$ are given. The particular function used is

$$
f(\lambda, x)=\lambda\left\{1+7(2 x-1)^{2} \ln \left[(2 x-1)^{2}\right]\right\} .
$$

It is useful to compare the $\delta_{n}$ and $\alpha_{n}$ in Tables I and II. In Table II the approach of $\delta_{n}, \alpha_{n} \rightarrow \delta, \alpha$ is much slower and less apparent (if at all). To check the forms (2.12) and (2.13) we note that (2.12) and (2.13) imply

$$
\begin{aligned}
& \delta_{n}=\delta\left[1-\frac{p \tau}{n}+O\left(\frac{1}{n^{2}}\right)\right], \\
& \alpha_{n}=\alpha\left[1-\frac{p \kappa}{n}+O\left[\frac{1}{n^{2}}\right]\right] .
\end{aligned}
$$

In Fig. 2, $\kappa$ versus $n$ is shown. Since (2.13) and (2.15) are expected to be correct only for large $n$, we have to consider

$$
\frac{\alpha_{n}}{\alpha}=1-\frac{p \kappa}{n}+\frac{A}{n^{2}}+\frac{B}{n^{3}}+\frac{C}{n^{4}},
$$

where the constants $\kappa, A, B$, and $C$ are to be determined via data fitting.

Curve 1 in Fig. 2 is obtained with the constants $A, B, C$ set to zero. Curve 2 has $B=C=0$, while $\kappa, A$ are determined by comparing $\alpha_{n-1}$ and $\alpha_{n}$. Curve 3 has $C=0$ while $\kappa, A$, and $B$ are determined by $\alpha_{n-1}, \alpha_{n}, \alpha_{n+1}$. Curve 4 gives $\kappa$ where $\kappa, A, B$, and $C$ are determined by $\alpha_{n-2}, \alpha_{n-1}, \alpha_{n}$, and $\alpha_{n+1}$. In Fig. 3, $\tau$ versus $n$ is shown, where a similar fitting has been performed. In Tables III and IV are the numerical $\kappa$ and $\tau$ obtained from Table II. If we assume the constants $\kappa$ and $\tau$ exist, then $\kappa \approx-0.44$ and $\tau \approx 0.37$. Next, let us consider some functions with the same $z, z=2$, but different values of $p$. To be specific, we consider

$$
f(x)=\lambda\left\{1-(2 x-1)^{2}\left[c\left|\ln (2 x-1)^{2}\right|^{p}+d\right]\right\},
$$

where the $(c, d, p)=\left(\frac{2}{5}, 1,2\right),(7,0,1),\left(1,0, \frac{1}{2}\right),\left(1,0,-\frac{1}{20}\right)$, $\left(1,0,-\frac{1}{10}\right),\left(1,0,-\frac{1}{5}\right),\left(\frac{3}{2}, 0,-1\right)$, and $(27,0,-2)$. In each case we extract the $\kappa$ and $\tau$ using formulas similar to Eq.

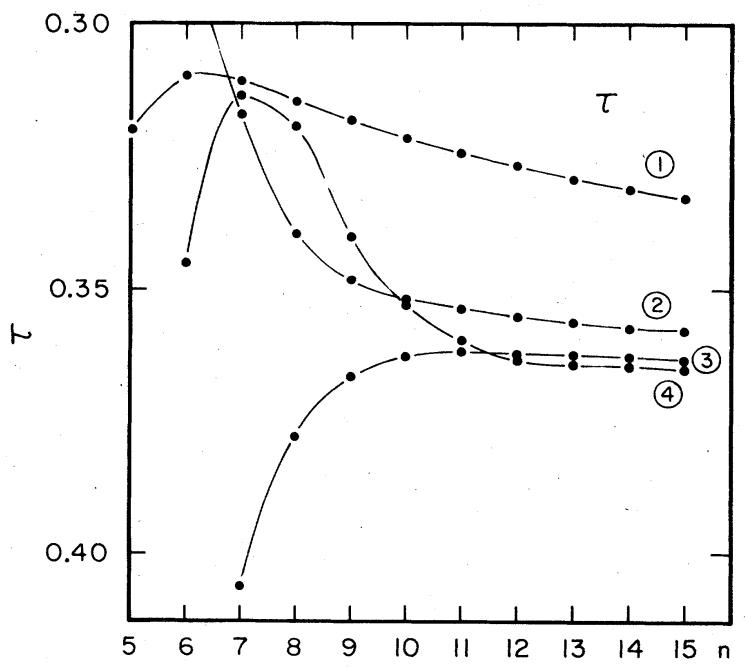

FIG. 3. Values of $\tau$ from Table IV, which are extracted from the numerical data of $\delta_{n}$ in Table II for the function (2.16). Curves $1,2,3$, and 4 correspond to $\tau_{1}, \tau_{2}, \tau_{3}$, and $\tau_{4}$ in Table IV. 
TABLE III. Value of $\kappa$ extracted from the $\alpha_{n}$ values in Table II. $\kappa_{1}$ is given by $\alpha_{n} / \alpha=1-\left(\kappa_{1} / n\right) ; \kappa_{2}$ is given by the fit $\alpha_{n} / \alpha=1-\left(\kappa_{2} / n\right)+\left(A / n^{2}\right)$ and its value can be obtained by comparing $\alpha_{n}$ and $\alpha_{n-1}$. Similarly, $\alpha_{n} / \alpha=1-\left(\kappa_{3} / n\right)+\left(A / n^{2}\right)+\left(B / n^{3}\right)$ and $\alpha_{n} / \alpha=1-\left(\kappa_{4} / n\right)+\left(A / n^{2}\right)+\left(B / n^{3}\right)+\left(C / n^{4}\right)$.

\begin{tabular}{rcccc}
\hline \hline$n$ & $\kappa_{1}$ & $\kappa_{2}$ & $\kappa_{3}$ & $\kappa_{4}$ \\
\hline 4 & -0.0979469 & -0.6007450 & -0.2952595 & -0.3502506 \\
5 & -0.1740677 & -0.4785508 & -0.2372735 & -0.1792875 \\
6 & -0.2114106 & -0.3981250 & -0.3211749 & -0.4330434 \\
7 & -0.2349432 & -0.3761393 & -0.3705955 & -0.4529632 \\
8 & -0.2524195 & -0.3747533 & -0.3993441 & -0.4568413 \\
9 & -0.2666193 & -0.3802179 & -0.4130603 & -0.4450646 \\
10 & -0.2786360 & -0.3867864 & -0.4200672 & -0.4387523 \\
11 & -0.2890180 & -0.3928375 & -0.4240696 & -0.4360767 \\
12 & -0.2981034 & -0.3980428 & -0.4267320 & -0.4356068 \\
13 & -0.3061305 & -0.4024565 & -0.4287155 & -0.4359885 \\
14 & -0.3132789 & -0.4062078 & -0.4302970 & -0.4366231 \\
15 & -0.3196883 & -0.4094197 & -0.4316048 & -0.4372717 \\
\hline \hline
\end{tabular}

(2.16). The results are shown in Figs. 4 and 5. Curve 4 in Figs. 2 and 3 are repeated in Figs. 4 and 5. The numerical data do provide good evidence that the constants $\kappa$ and $\tau$ exist for both positive and negative real $p$, with $\kappa \approx-0.445$ and $\tau \approx 0.370$ where errors are of the order of a few percent.

It is very difficult to improve the accuracy of $\kappa$ and $\tau$ via straight numerical computation. This is because the computation time increases exponentially with $n$ while the accuracy increases only like inverse powers of $n$. For large $|p|$ the numerical determinations of $\kappa$ and $\tau$ become worse.

In the rest of this paper we shall use the renormalization-group method

(1) to verify the form (2.12) and (2.13) for all positive integer $p$,

(2) to determine $\kappa$ and $\tau$ to better precisions,

(3) to elucidate the functional relations among $\alpha, \delta, \kappa$, and $\tau$, and

(4) to extend the result to the general form

$$
\begin{aligned}
f(x)-f\left(x_{c}\right) \approx & \left|x-x_{c}\right|^{z}|\ln | x-x_{c}||^{p} \\
& \times|\ln | \ln \left|x-x_{c}\right|||^{q} \ldots
\end{aligned}
$$

by a combination of analytical and numerical arguments.

Feigenbaum's analysis of the iterated maps uses the renormalization-group method. In fact, his work provides the starting point of our investigation. After the renormalization-group analysis, we derive the universal quantitative properties for the general case.

\section{THE $p=1$ CASE}

In this section we consider only the $p=1$ case. A brief review of Feigenbaum's renormalization-group analysis ${ }^{2}$ is followed by its generalization to the case relevant to the $p=1$ case. Equation (2.15) [hence (2.12) and (2.13)] shall be derived analytically for the $p=1$ case. Some of the details are relegated to the three appendixes.

\section{A. Review}

For some general function $f(\lambda, x)$, its $m$ th iteration is given by

$$
f^{m}(\lambda, x)=f(\lambda, f(\lambda, f(\cdots))) .
$$

Without loss of generality we can set the extremum at the origin, i.e., $x_{c}=0$. Let

TABLE IV. Value of $\tau$ extracted from the $\delta_{n}$ values in Table II. $\tau_{1}, \tau_{2}, \tau_{3}$, and $\tau_{4}$ are obtained in a way similar to that for $\kappa$ in Table III.

\begin{tabular}{rcrcc}
\hline \hline$n$ & $\tau_{1}$ & \multicolumn{1}{c}{$\tau_{2}$} & $\tau_{3}$ & $\tau_{4}$ \\
\hline 4 & 0.3717936 & -0.1344284 & 0.4803375 & 0.5546635 \\
5 & 0.3197304 & 0.1114780 & 0.5525065 & 0.6246754 \\
6 & 0.3095233 & 0.2584875 & 0.4633065 & 0.3443733 \\
7 & 0.3105924 & 0.3170072 & 0.4069723 & 0.3130820 \\
8 & 0.3142057 & 0.3394985 & 0.3777015 & 0.3191597 \\
9 & 0.3179593 & 0.3479880 & 0.3663880 & 0.3399899 \\
10 & 0.3213301 & 0.3516680 & 0.3626793 & 0.3527896 \\
11 & 0.3242701 & 0.3536701 & 0.3618739 & 0.3594577 \\
12 & 0.3268341 & 0.3550374 & 0.3620174 & 0.3624956 \\
13 & 0.3290862 & 0.3561112 & 0.3624216 & 0.3639039 \\
14 & 0.3310809 & 0.3570127 & 0.3628632 & 0.3646293 \\
15 & 0.3328617 & 0.3577928 & 0.3632784 & 0.3650778 \\
\hline \hline
\end{tabular}




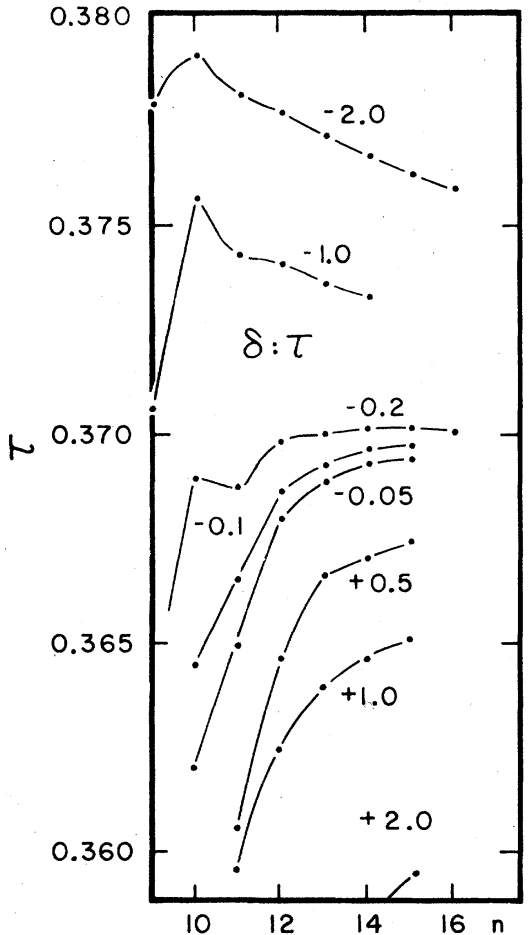

FIG. 4. Value of $\tau$ extracted from functions with different $p$ values. The $p$ value for each case is displayed next to the curve; all the curves correspond to $\tau_{4}$.

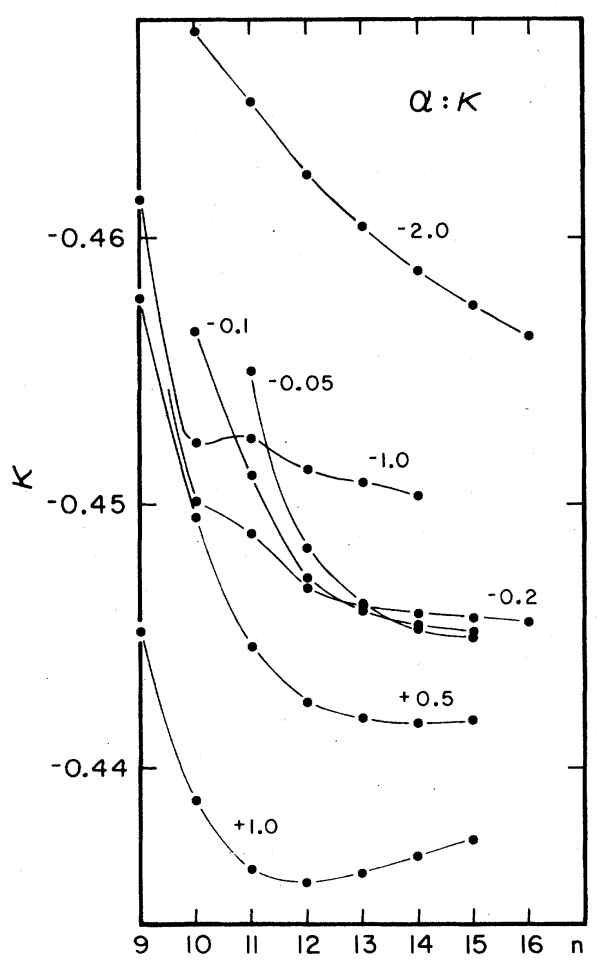

FIG. 5. Value of $\kappa$ extracted from functions with different $p$ values. The $p$ value for each case is displayed next to the curve. All the curves correspond to $\kappa_{4}$.

$$
\widetilde{g}_{n}(x) \equiv(-1)^{n} \beta_{n} f^{2^{n}}\left[\lambda_{\infty}, \frac{x}{\beta_{n}}\right],
$$

where $\lambda_{\infty}$ is the $\lambda$ that has a stable orbit with an infinite period. This is the critical value beyond which is chaos. For the sake of simplicity we assume $\widetilde{g}_{n}(x)$ to be symmetric, i.e., $\widetilde{g}_{n}(x)=\widetilde{g}_{n}(-x)$. Replacing $x$ by $\beta_{n} x$ we have

$$
\frac{1}{\beta_{n}} \widetilde{g}_{n}\left(\beta_{n} x\right)=(-1)^{n} f^{2^{n}}\left(\lambda_{\infty}, x\right) \text {. }
$$

Then, using (3.3), we obtain

$$
\frac{-1}{\beta_{n+1}} \widetilde{g}_{n+1}\left(\beta_{n+1} x\right)=\frac{1}{\beta_{n}} \widetilde{g}_{n}\left(\widetilde{g}_{n}\left(\beta_{n} x\right)\right)
$$

or, replacing $x$ by $x / \beta_{n+1}$,

$$
I\left[\alpha_{n}, \widetilde{g}_{n}\right] \equiv-\alpha_{n} \widetilde{g}_{n}\left(\widetilde{g}_{n}\left(x / \alpha_{n}\right)\right)=\widetilde{g}_{n+1}(x),
$$

where $\alpha_{n}=\beta_{n+1} / \beta_{n}$. If the limit $n \rightarrow \infty$ exists for (3.5) then $\widetilde{g}_{n}(x) \rightarrow g(x), \alpha_{n} \rightarrow \alpha$ as $n \rightarrow \infty$, and

$$
I[\alpha, g]=-\alpha g(g(x / \alpha))=g(x),
$$

where $g(x)$ is the universal function. Since the normalization is not fixed, we can define

$$
g(0)=1
$$

which gives $g(1)=-1 / \alpha$. Functionally, $g(x)$ can be written as

$$
g_{z}(x)=1+g_{1}|x|^{2}+\cdots,
$$

where the coefficients as well as $\alpha$ can be determined numerically by using Eq. (3.6). From (3.6) and (3.8) it is easy to obtain

$$
g_{z}^{\prime}(1)=-\alpha^{z-1} \text {. }
$$

For large $n$ we write

$$
\widetilde{g}_{n}(x)=g(x)+\sum_{i} c_{n}^{(i)} E_{i}(x),
$$

where $E_{i}(x)$ are a set of functions to be determined. For large $n$ the coefficients $c_{n}^{(i)}$ are expected to be small. Substituting Eq. (3.10) into Eq. (3.5) and using Eq. (3.6), we have

$$
\sum_{i} c_{n+1}^{(i)} E_{i}(x)=\sum_{i} c_{n}^{(i)} L\left[\alpha_{n}, E_{i}\right]+\cdots,
$$

where the operator $L\left[\alpha_{n}, E_{i}\right]$, the linearized operator of $I\left[\alpha_{n}, \widetilde{g}_{n}\right]$, is defined by

$$
\begin{aligned}
L\left[\alpha_{n}, E_{i}\right] \equiv-\alpha_{n}\left[g_{z}^{\prime}\left(g_{z}\left(\frac{x}{\alpha_{n}}\right)\right] E_{i}\left(\frac{x}{\alpha_{n}}\right)\right. \\
\left.+E_{i}\left(g_{z}\left(\frac{x}{\alpha_{n}}\right)\right]\right)
\end{aligned}
$$

and higher-order terms are neglected, and

$$
g_{z}^{\prime}(x)=\frac{d g_{z}(x)}{d x} .
$$

Since $L$ is a linear operator, its eigenvalues and eigenvec- 
tors can be determined (numerically or analytically). In the case without logarithms, it is found that there is one eigenvector with eigenvalue larger than one; its value is $\delta(\delta>2)$ :

$$
L[\alpha, h]=\delta h(x) .
$$

In the renormalization-group language, $h(x)$ is a relevant operator. There is also an eigenvector with eigenvalue 1 , i.e.,

$$
L[\alpha, t]=t(x) .
$$

Eigenvectors with eigenvalue 1 are known as marginal operators. In this case it is easy to show that

$$
t(x)=g(x)-x g^{\prime}(x) \text {. }
$$

Because of Eq. (3.15), $t(x)$ is also known as a redundant operator. To see its meaning, let us consider $\mu g(x / \mu)$, which clearly satisfies (3.6). For $\mu=1+\epsilon, \epsilon$ small, we have

$$
\begin{aligned}
R[\epsilon, g(x)] & \equiv(1+\epsilon) g(x /(1+\epsilon)) \\
& =g(x)+\epsilon t(x)+\frac{\epsilon^{2} x^{2} g^{\prime \prime}(x)}{2}+O\left(\epsilon^{3}\right) .
\end{aligned}
$$

Therefore $t(x)$ is the rescaling operator. In the absence of logarithms, $\alpha_{n} \approx \alpha$, and (3.11) can be written as

$$
\begin{aligned}
u_{n+1} h(x)+ & w_{n+1} t(x)+\sum_{j} c_{n+1}^{(j)} k_{j}(x) \\
& =\delta u_{n} h(x)+w_{n+1} t(x)+\sum_{j} e^{(j)} c_{n}^{(j)} k_{j}(x)+\cdots,
\end{aligned}
$$

where $k_{j}$ is an eigenvector with eigenvalue $e^{(j)}$, where $e^{(j)}<1 . k_{j}(x)$ is known as an irrelevant operator since its coefficient $c_{n}^{(j)} \approx\left(e^{(j)}\right)^{n}$ will in general decrease geometrically and become negligible rapidly. Equation (3.17) is valid because $h(x), t(x)$, and $k_{j}(x)$ form a complete set of eigenfunctions. To leading order, we have, from (3.17),

$$
\begin{aligned}
& u_{n+1}=\delta u_{n}+\cdots, \\
& w_{n+1}=w_{n}+\cdots .
\end{aligned}
$$

If we choose a normalization such that for some $n$, say $n_{0}$, such that $w_{n_{0}}=0$, then $w_{n}=0$ for $n \geq n_{0}$, and, for large $n, u_{n} \simeq c \delta^{n}$, for some constant $c$. This means the approach to chaos is geometric. $\delta$ in Eq. (3.18) is the Feigenbaum constant.

\section{B. The $p=1$ operator}

In the case where $\widetilde{g}_{n}(x)$ is expected to have a logarithmic factor of the form $|x|^{z} \ln \left(x^{2}\right)$, we expect

$$
\widetilde{g}_{n}(x)=g(x)+u_{n} h(x)+w_{n} t(x)+v_{n} s(x)+\cdots,
$$

where $s(x) \approx|x|^{2} \ln \left(x^{2}\right)+\cdots$. For $x \rightarrow 0$, this term should dominate and, using (3.9),

$$
\begin{aligned}
L[\alpha, s] & \approx-\alpha\left[g^{\prime}(1) s(x / \alpha)+\cdots\right] \\
& \approx|x|{ }^{2} \ln \left(x^{2}\right)+\cdots,
\end{aligned}
$$

indicating that the corresponding eigenvalue is 1 and hence $s(x)$ is a marginal operator. However, $s(x)$ is not an eigenvector, as we now demonstrate. To present the $z$ dependence more explicitly, let us write (3.6) as

$$
I[\alpha, g]=-\alpha_{z} g_{z}\left(g_{z}\left(x / \alpha_{z}\right)\right)=g_{z}(x) .
$$

Similarly, for small $\epsilon$, we have

$$
-\alpha_{z+\epsilon} g_{z+\epsilon}\left(g_{z+\epsilon}\left(x / \alpha_{z+\epsilon}\right)\right)=g_{z+\epsilon}(x),
$$

where

$$
\begin{aligned}
& g_{z+\epsilon}(x)=g_{z}(x)+\epsilon \frac{\partial g_{z}}{\partial z}(x)+\cdots, \\
& \alpha_{z+\epsilon}=\alpha_{z}+\epsilon \frac{\partial \alpha_{z}}{\partial z}+\cdots .
\end{aligned}
$$

From (3.8) we have

$$
\begin{aligned}
\widetilde{s}(x) & \equiv \frac{\partial g_{z}}{\partial z}(x) \\
& =\left(\frac{\partial g_{1}}{\partial z}\right)|x|^{z}+\frac{g_{1}}{2}|x|^{z} \ln \left(x^{2}\right)+\cdots .
\end{aligned}
$$

Substituting (3.22) - (3.24) into (3.21) and using (3.20), we obtain, for terms with the leading power in $\epsilon$,

$$
\begin{array}{r}
-\alpha\left[g_{z}^{\prime}\left[g_{z}\left(\frac{x}{\alpha}\right)\right] \widetilde{s}(x)+\widetilde{s}\left[g_{z}\left(\frac{x}{\alpha}\right)\right]\right. \\
-\frac{1}{\alpha^{2}}\left(\frac{\partial \alpha_{z}}{\partial z}\right) g_{z}^{\prime}\left[g_{z}\left(\frac{x}{\alpha}\right)\right] x g_{z}^{\prime}\left(\frac{x}{\alpha}\right) \\
\left.+\frac{1}{\alpha}\left(\frac{\partial \alpha_{z}}{\partial z}\right) g_{z}\left(g_{z}\left(\frac{x}{\alpha}\right)\right]\right]=\widetilde{s}(x) .
\end{array}
$$

Using (3.15) we obtain

$$
L[\alpha, s]=s(x)+t(x),
$$

where

$$
\begin{aligned}
s(x) & =-\alpha_{z}\left(\frac{\partial \alpha_{z}}{\partial z}\right)^{-1} \tilde{s}(x) \\
& =-\alpha_{z}\left(\frac{\partial \alpha_{z}}{\partial z}\right)^{-1} \frac{\partial g_{z}(x)}{\partial z} .
\end{aligned}
$$

Equations (3.14) and (3.25) show that the operator $L$ is not symmetric, so that it takes the Jordan canonical form. $s(x)$ is the new marginal operator for $p=1$. We observe that if $s(x)$ is a solution of (3.25), then so is $s(x)-r t(x)$ for any constant $r$. We shall come back to this point later.

\section{The renormalization-group equation}

Using (3.5) and (3.19) and Taylor expansion, it is straightforward to obtain, for $n$ large and small coefficients $u_{n}$, $v_{n}$, $w_{n}$, 


$$
\begin{aligned}
g(x)+ & u_{n+1} h(x)+w_{n+1} t(x)+v_{n+1} s(x)+\cdots=I\left[\alpha_{n}, g_{n}\right] \\
= & -\alpha_{n} \widetilde{g}_{n}\left(\widetilde{g}_{n}\left(x / \alpha_{n}\right)\right) \\
= & -\alpha_{n} g\left(g\left(x / \alpha_{n}\right)\right)+u_{n} L\left[\alpha_{n}, h\right]+w_{n} L\left[\alpha_{n}, t\right]+v_{n} L\left[\alpha_{n}, s\right]+\frac{u_{n}^{2}}{2} Q\left[\alpha_{n}, h, h\right] \\
& +u_{n} w_{n} Q\left[\alpha_{n}, h, t\right]+u_{n} v_{n} Q\left[\alpha_{n}, h, s\right]+\frac{w_{n}^{2}}{2} Q\left[\alpha_{n}, t, t\right]+w_{n} v_{n} Q\left[\alpha_{n}, t, s\right]+\frac{v_{n}^{2}}{2} Q\left[\alpha_{n}, s, s\right]+\cdots,
\end{aligned}
$$

where terms from the irrelevant operators are neglected on both sides. The terms that are cubic in the coefficients $u_{n}, v_{n}, w_{n}$ are ignored in the right-hand side. The operator $L$ is defined in (3.12) and the operator $Q$ is defined as follows:

$$
\begin{aligned}
Q\left[\alpha_{n}, t, h\right]=Q\left[\alpha_{n}, h, t\right]=-\alpha_{n} & {\left[g^{\prime \prime}\left[g\left(\frac{x}{\alpha_{n}}\right)\right] h\left[\frac{x}{\alpha_{n}}\right) t\left(\frac{x}{\alpha_{n}}\right)\right.} \\
& \left.+h^{\prime}\left[g\left(\frac{x}{\alpha_{n}}\right)\right] t\left(\frac{x}{\alpha_{n}}\right)+h\left(\frac{x}{\alpha_{n}}\right) t^{\prime}\left(g\left(\frac{x}{\alpha_{n}}\right)\right]\right]
\end{aligned}
$$

and similarly for the other $Q$ operators. Let us consider the various terms on the right-hand side of Eq. (3.29). By using (3.6) the first term can be written as

$$
\begin{aligned}
-\alpha_{n} g\left(g\left(x / \alpha_{n}\right)\right)= & \left(\alpha_{n} / \alpha\right) g\left(\alpha x / \alpha_{n}\right) \\
= & R\left[\epsilon_{n}, g(x)\right] \\
= & g(x)+\epsilon_{n} t(x) \\
& +\epsilon_{n}^{2} \frac{x^{2}}{2} g^{\prime \prime}(x)+O\left(\epsilon_{n}^{3}\right),
\end{aligned}
$$

where (3.16) is used. $\epsilon_{n} \equiv-1+\alpha_{n} / \alpha$ is taken to be small for large $n$.

Using (3.13) on the second term on the right-hand side (rhs) of (3.27), we have

$$
\begin{aligned}
L\left[\alpha_{n}, h\right] & =\delta\left(1+\epsilon_{n}\right) h\left(x /\left(1+\epsilon_{n}\right)\right) \\
& =\delta R\left[\epsilon_{n}, h(x)\right] \\
& =\delta\left\{h(x)+\epsilon_{n}\left[h(x)-x h^{\prime}(x)\right]\right\}
\end{aligned}
$$

and similarly for the next two terms. The goal is to express the terms in (3.27) in terms of $g(x), h(x), t(x)$, $s(x)$, and the irrelevant operators. $h(x), t(x), s(x)$, and the irrelevant operators should form a complete basis so that we can write

$$
\begin{aligned}
& \frac{x^{2} g^{\prime \prime}(x)}{2}=d_{1} h(x)+e_{1} t(x)+f_{1} s(x)+\cdots, \\
& h(x)-x h^{\prime}(x)=d_{2} h(x)+e_{2} t(x)+f_{2} s(x)+\cdots, \\
& t(x)-x t^{\prime}(x)=d_{3} h(x)+e_{3} t(x)+f_{3} s(x)+\cdots, \\
& s(x)-x s^{\prime}(x)=d_{4} h(x)+e_{4} t(x)+f_{4} s(x)+\cdots,
\end{aligned}
$$

where $d_{i}, e_{i}$, and $f_{i}$ are coefficients to be determined. The irrelevant operators are not written out explicitly. If we keep terms only up to second power in $u_{n}, v_{n}, w_{n}$ and $\epsilon_{n}$ in Eq. (3.27), then $Q\left[\alpha_{n}, h, h\right] \simeq Q[\alpha, h, h]$, $Q\left[\alpha_{n}, h, t\right]=Q[\alpha, h, t]$, etc., so that

$$
\begin{aligned}
& Q[\alpha, h, h]=a_{1} h(x)+b_{1} t(x)+c_{1} s(x)+\cdots, \\
& Q[\alpha, h, t]=a_{2} h(x)+b_{2} t(x)+c_{2} s(x)+\cdots, \\
& Q[\alpha, t, t]=a_{3} h(x)+b_{3} t(x)+c_{3} s(x)+\cdots, \\
& Q[\alpha, h, s]=a_{4} h(x)+b_{4} t(x)+c_{4} s(x)+\cdots, \\
& Q[\alpha, s, t]=a_{5} h(x)+b_{5} t(x)+c_{5} s(x)+\cdots, \\
& Q[\alpha, s, s]=a_{6} h(x)+b_{6} t(x)+c_{6} s(x)+\cdots,
\end{aligned}
$$

where $a_{i}, b_{i}$, and $c_{i}$ are coefficients to be determined. We observe that some of the coefficients in (3.34) must be trivially zero. $g(x), h(x), t(x)$, and their derivatives are simple polynomials in $x$ (and/or fractional powers of $x$ ). Hence, $f_{1}=f_{2}=f_{3}=c_{1}=c_{2}=c_{3}=0$. (Other properties of the coefficients shall be discussed later.) Now we can equate the coefficients of $h(x), t(x)$, and $s(x)$ [in Eqs. (3.27)-(3.29)] to obtain these coupled equations,

$$
\begin{aligned}
u_{n+1}= & \delta u_{n}+\frac{a_{1}}{2} u_{n}^{2}+a_{2} u_{n} w_{n}+\frac{a_{3}}{2} w_{n}^{2} \\
& +a_{4} u_{n} v_{n}+a_{5} w_{n} v_{n}+\frac{a_{6}}{2} v_{n}^{2}+\delta d_{2} u_{n} \epsilon_{n} \\
& +d_{3} \epsilon_{n} w_{n}+\left(d_{3}+d_{4}\right) \epsilon_{n} v_{n}+\cdots, \\
w_{n+1}= & w_{n}+v_{n}+\epsilon_{n}+\frac{b_{1}}{2} u_{n}^{2}+b_{2} u_{n} w_{n}+\frac{b_{3}}{2} w_{n}^{2} \\
& +b_{4} u_{n} v_{n}+b_{5} w_{n} v_{n}+\frac{b_{6}}{2} v_{n}^{2}+e_{2} \delta u_{n} \epsilon_{n}+e_{3} \epsilon_{n} w_{n} \\
& +\left(e_{3}+e_{4}\right) \epsilon_{n} v_{n}+e_{1} \epsilon_{n}^{2}+\cdots
\end{aligned}
$$

$v_{n+1}=v_{n}+c_{4} u_{n} v_{n}+c_{5} w_{n} v_{n}+\frac{c_{6}}{2} v_{n}^{2}+f_{4} \epsilon_{n} v_{n}+\cdots$,

where the $v_{n}$ term appearing in (3.36) is due to (3.25). 
These three coupled equations form the set of renormalization-group equations. To simplify this set of equations, we now make a number of observations.

(1) $t(x)$ is a simple function of $g(x)$, as given by (3.15); also, $t^{\prime}(x)=-x g^{\prime \prime}(x)$. Hence, from (3.33), we obtain

$$
\begin{aligned}
& d_{3}=2 d_{1}, \\
& e_{3}=2 e_{1}+1 .
\end{aligned}
$$

(2) Both $w_{n}$ and $\epsilon_{n}$ are related to rescaling. Hence for each $n$, we can choose $\epsilon_{n}$ such that the rhs of (3.36) vanishes:

$$
\epsilon_{n}=-v_{n}+\cdots \text {. }
$$

Then $w_{n}$ can be set to zero for all $n$,

$$
w_{n}=0 \text {. }
$$

This simplifies the other two equations as well.

(3) $u_{n}$ is taken to be much smaller than $v_{n}$. This is necessary since further iteration will increase $u_{n}$ geometrically (i.e., $u_{n+m} \sim \delta^{m} u_{n}$ where $\delta>1$ ), while the renormalization-group equations assume a perturbative expansion in $u_{n}, v_{n}, w_{n}$, etc. Clearly, the validity of the renormalization-group equations break down when $u_{n+m} \sim 1$ for some $m$ (see Ref. 5 for a detailed argument). Hence, to the approximation we need, (3.35)-(3.37) reduce to a much simpler set of equations,

$$
\begin{aligned}
& u_{n+1}=\delta u_{n}+\left(a_{4}-d_{2} \delta\right) u_{n} v_{n}, \\
& v_{n+1}=v_{n}+\left[\frac{c_{6}}{2}-f_{4}\right] v_{n}^{2}, \\
& \alpha_{n}=\alpha\left(1+\epsilon_{n}\right)=\alpha\left(1-v_{n}\right),
\end{aligned}
$$

where (3.41) follows from the definition of $\epsilon_{n}$.

(4) We can now solve (3.40) to obtain, for large $n$,

$$
-\epsilon_{n} \approx v_{n} \approx \frac{-1}{\left[\frac{c_{6}}{2}-f_{4}\right]_{n}}
$$

Equations (3.40)-(3.42) verify Eq. (2.15) for the $p=1$ case. Comparing (3.41) and (3.42) with (2.15) for $p=1$, we obtain

$$
\kappa=-\left(\frac{c_{6}}{2}-f_{4}\right)^{-1}
$$

Substituting (3.42) into (3.39), we obtain

$$
u_{n+1}=\delta\left[1+\frac{\left(a_{4}-d_{2} \delta\right)_{\kappa}}{n \delta}\right] u_{n} .
$$

Comparing this with (2.15) and (3.18), we obtain

$$
\tau=-\frac{\kappa}{\delta}\left(a_{4}-d_{2} \delta\right)
$$

Hence we have shown analytically the asymptotic form (2.15) for the $p=1$ case since $\kappa$ and $\tau$ are independent of n. Equations (2.12) and (2.13) immediately follow from (2.15). The coefficients $c_{6} f_{4}, a_{4}$, and $d_{2}$ can be determined numerically. This will be done in Sec. IV. Equa- tions (3.43) and (3.44) provide a renormalization-group method calculation of the constants $\kappa$ and $\tau$.

(5) There is one very important technical point that we have ignored so far. As mentioned earlier, if $s(x)$ is a solution of (3.25), then so is $s(x)-r t(x)$ for any constant $r$. It is clear from (3.33) and (3.34) that a change of $s(x)$ will cause corresponding changes in some of the coefficients. This is discussed in Appendix A. In particular, under $\quad s(x) \rightarrow s(x)-r t(x), \quad a_{4} \rightarrow a_{4}-r a_{2}$ and $c_{6} \rightarrow c_{6}$ $-2 r c_{5}$ while $f_{4}$ and $d_{2}$ remain invariant, so that Eq. (3.43) becomes

$$
\kappa=-\left(\frac{c_{6}}{2}-r c_{5}-f_{4}\right]^{-1}
$$

and Eq. (3.44) becomes

$$
\tau=-\frac{\kappa}{\delta}\left(a_{4}-r a_{2}-d_{2} \delta\right)
$$

This will imply that $\kappa$ and $\tau$ are independent of $r$ if and only if $a_{2}$ and $c_{5}$ are exactly zero for all choices of $r$. This turns out to be precisely the case. The proof follows from the properties of the operators $I$ and $R$ and is presented in Appendix B. Here let us summarize the results. Independent of $r$, we obtain

$$
\begin{aligned}
& a_{2}=b_{3}=c_{5}=0, \\
& a_{3}=(1-\delta) d_{3}, \\
& a_{5}=d_{3}+(1-\delta) d_{4}, \\
& b_{2}=(1-\delta) e_{2}, \\
& b_{5}=2\left(e_{1}+1\right),
\end{aligned}
$$

where we also have used the fact that

$$
f_{4}=1-z
$$

which follows easily from an examination of (3.33) and Appendix C.

(6) It is clear, to calculate the constants $\kappa$ and $\tau$, that we must first determine the functions $g(x), h(x), t(x)$, and $s(x)$ numerically; then we evaluate the coefficients $a_{4}, c_{6}$, and $d_{2}$. Here we want to show that $c_{6}$ and hence $\kappa$ can be calculated in terms of the coefficients in the eigenfunction $s(x)$ and the universal function $g(x)$. In Appendix $C$ we show that

$$
\kappa^{-1}=-\left(\frac{c_{6}}{2}+z-1\right)=-\frac{2 \beta_{1} \ln \alpha}{g_{1}},
$$

where $\beta_{1}$ is the coefficient of $|x|^{z} \ln x^{2}$ in $s(x)$, i.e.,

$$
s(x)=\beta_{1}|x|^{2} \ln \left(x^{2}\right)+\cdots,
$$

and $g_{1}$ is the coefficient of $|x|^{z}$ in $g(x)$ as given in (3.8).

(7) It is simple to see how Feigenbaum's result can be recovered from (3.35)-(3.37). In the absence of logarithmic factors, $v_{n}=0$. Then $\epsilon_{n}$ and $w_{n}$ can both be taken to be zero and we are left with $u_{n+1}=\delta u_{n}$ only. This is the renormalization-group equation in Feigenbaum's case. It clearly implies that $\delta$ is independent of $n$ and hence a universal constant. It is the presence of the $v_{n}$ terms in 
(3.36) that forces us to have a nontrivial additional rescaling $\epsilon_{n}$. With this, even if $w_{n}=0$ for some value of $n$, $w_{n+1}=v_{n}+\cdots$ does not vanish unless $v_{n}=0$. Further iteration renders Eq. (3.36) nontrivial again. This rescaling after each operation $I\left[\alpha, \widetilde{g}_{n}\right]$ is analogous to the anomalous dimension of the wave-function renormalization that is present in quantum-field theory, while the usual rescaling by factor $\alpha$ corresponds to the canonical dimension.

This completes our renormalization-group analysis for the $p=1$ case. To summarize, Eqs. (3.43) and (3.47) give $\kappa$ and $\tau$ in terms of some coefficients. These coefficients, namely $\beta_{1}, g_{1}, a_{4}, d_{2}$ as well as $\alpha$ and $\delta$, are to be determined numerically. Alternatively, we can use (3.44) instead of (3.47) to determine $\kappa$.

\section{GENERALIZATION TO THE $p=$ POSITIVE INTEGER CASE}

\section{A. The Jordan canonical form}

Here we shall consider the $p=$ positive integer case and the functional relation among $\alpha, \delta, \kappa$, and $\tau$. First let us examine the functional space for $h(x), t(x)$, and $s(x)$. From now on, $s(x)$ in Sec. III shall be referred to as $s_{p=1}(x)$ or simply $s_{1}(x)$.

$A$ natural choice of the functional space to which $h(x), t(x)$ belong is the set of linear combinations of powers of $x$, that is, $|x|^{z n} x^{2 m}, n=0,1,2, \ldots$ and $m=0,1,2, \ldots$ This means $h(x)=1+h_{1}|x|^{2}$ $+h_{2}|x|^{z+2}+h_{3}|x|^{z+4}+\cdots$ and similarly for $t(x)$. [Here the normalization of $h(x), t(x)$ is taken to be $h(0)=t(0)=1$.] Let us refer to this as the functional space $F_{z}(p=0)$. To obtain the additional eigenvalue 1 for $s_{1}(x)$, we must enlarge the functional space to include the logarithmic factors. An examination of (3.26) clearly indicates that terms like $\ln \left(x^{2}\right)\left(\beta_{1}|x|^{2}+\right.$ terms with higher powers of $|x|$ must be included in addition to terms in $F_{z}(p=0)$. In order to define the operator $Q[\alpha]$ in (3.34), we see that terms like $\left[\ln \left(x^{2}\right)\right]^{n}\left(|x|^{2 z}+\right.$ terms with higher powers of $|x|), n=0,1,2$, must also be included. If we want to keep higher-order terms in the renormalization-group equation (3.29), it is easy to see that a natural choice of the functional space to which $h(x), t(x)$, and $s_{1}(x)$ belong is the set of the linear combinations of

$$
\begin{aligned}
& {\left[|x|^{z n}\left|\ln \left(x^{2}\right)\right|^{m}\right] x^{2 k} \text { with } n=0,1,2, \ldots,} \\
& \quad m=0,1,2, \ldots, n, \text { and } k=0,1,2, \ldots .
\end{aligned}
$$

This is referred to as $F_{z}(p=1)$,

$$
F_{z}(p=0) \subset F_{z}(p=1) \text {. }
$$

Now let us consider the case of positive integer $p$. A similar argument shows that for positive integer $p, F_{z}(p)$ is the set of linear combinations of $|x|^{z n}\left|\ln x^{2}\right|^{m} x^{2 k}$ where $n=0,1,2, \ldots ; m=0,1,2, \ldots, p n ; k=0,1,2, \ldots . \quad$ In terms of the operator $L$ in (3.12), there is one eigenvalue of unity if the functional space is restricted to $F_{z}(p=0)$, as in the original work of Feigenbaum. In Sec. III we found two degenerate eigenvalues of unity because the functional space has been enlarged to $F_{z}(p=1)$. In general, there will be $p+1$ degenerate eigenvalues of unity (hence $p+1$ marginal operators) for the functional space $F_{z}(p=$ positive integer). It is straightforward to show that they appear in the Jordan canonical form

$$
\begin{gathered}
L[\alpha, t]=t, \\
L\left[\alpha, s_{1}\right]=s_{1}+t, \\
L\left[\alpha, s_{2}\right]=s_{2}+s_{1}, \\
\vdots \\
L\left[\alpha, s_{i+1}\right]=s_{i+1}+s_{i}, \\
\vdots \\
L\left[\alpha, s_{p}\right]=s_{p}+s_{p-1} .
\end{gathered}
$$

To illustrate this, let us consider the $s_{2}(x)$ case

$$
s_{2}(x)=\beta_{2}|x|^{z}\left[\ln \left(x^{2}\right)\right]^{2}+\eta_{1}|x|^{z} \ln \left(x^{2}\right)+\cdots,
$$

where the dominant terms (as $x \rightarrow 0$ ) are kept. Using (3.9) we find that

$$
\begin{aligned}
L\left[\alpha, s_{2}\right]= & \beta_{2}|x|^{z}\left[\ln \left(x^{2}\right)\right]^{2} \\
& +\left(\eta_{1}-4 \beta_{2} \ln \alpha\right)|x|^{z} \ln \left(x^{2}\right)+\cdots
\end{aligned}
$$

so that consistency requires $L\left[\alpha, s_{2}\right]=s_{2}+s_{1}$ and

$$
-4 \beta_{2} \ln \alpha=\beta_{1}
$$

where $\beta_{1}$ is the coefficient in $s_{1}(x)$ as given in (3.48). Knowing $\beta_{1}$, the normalization and the most important coefficient in $s_{2}(x)$ are determined. (On the other hand, $\eta_{1}$ is arbitrary. It depends on the choice of basis. If we want, we can choose it to be zero.) In fact, for, $m=1,2, \ldots, p$,

$$
s_{m}(x)=\beta_{m}|x|^{z}\left[\ln \left(x^{2}\right)\right]^{m}+\cdots
$$

we have

$$
-m \beta_{m} \ln \left(\alpha^{2}\right)=\beta_{m-1}
$$

or

$$
\beta_{m}=\frac{(-2 \ln \alpha)^{1-m}}{m !} \beta_{1}
$$

\section{B. The renormalization-group equation}

If the function to be considered has its extremum with the behavior $|x|^{z}\left|\ln \left(x^{2}\right)\right|^{m}$, then the corresponding $g_{n}(x)$ can be written as

$$
g_{n}(x)=g(x)+u_{n} h(x)+w_{n} t(x)+\sum_{i=1}^{m} v_{n}^{(i)} s_{i}(x)
$$

Substituting this into (3.5) and following the steps similar to those in Sec. III, we obtain the following set of renormalization-group equations, for large $n$ : 


$$
\begin{aligned}
u_{n+1}= & \delta u_{n}+a_{4} u_{n} v_{n}^{(1)}+\delta d_{2} \epsilon_{n} u_{n}+\cdots, \\
w_{n+1}= & w_{n}+v_{n}^{(1)}+\epsilon_{n}+\cdots, \\
v_{n+1}^{(1)}= & v_{n}^{(1)}+v_{n}^{(2)}+\frac{c_{6}}{2} v_{n}^{(1)^{2}}+f_{4} \epsilon_{n} v_{n}^{(1)}+\cdots, \\
v_{n+1}^{(2)}= & v_{n}^{(2)}+v_{n}^{(3)}+B^{(2)} v_{n}^{(1)} v_{n}^{(2)} \\
& +C^{(2)} \epsilon_{n} v_{n}^{(2)}+\cdots, \\
& \vdots \\
v_{n+1}^{(m-1)}= & v_{n}^{(m-1)}+v_{n}^{(m)}+B^{(m-1)} v_{n}^{(1)} v_{n}^{(m-1)} \\
& +C^{(m-1)} \epsilon_{n} v_{n}^{(m-1)}+\cdots, \\
v_{n+1}^{(m)}= & v_{n}^{(m)}+B^{(m)} v_{n}^{(1)} v_{n}^{(m)}+C^{(m)} \epsilon_{n} v_{n}^{(m)}+\cdots,
\end{aligned}
$$

where $B^{(i)}$ and $C^{(i)}(i=2,3, \ldots)$ are defined by the following equations:

$$
Q\left[\alpha, s_{i}, s_{1}\right]=B^{(i)} s_{i}(x)+\cdots
$$

and

$$
s_{i}(x)-x s_{i}^{\prime}(x)=C^{(i)} s_{i}(x)+\cdots
$$

Here the terms not displayed shall turn out to be small compared to the terms shown. Again, we can choose $\epsilon_{n}=-v_{n}^{(1)}$, so that $w_{n}=0$ for all $n$. Let us define

$$
A^{(i)}=B^{(i)}-C^{(i)}
$$

Before solving the renormalization-group equations to the order that is of interest to us, first let us show that

$$
A^{(m)}=A^{(m-1)}=A^{(i)}=A^{(1)}=\frac{c_{6}}{2}+z-1 .
$$

The rhs follows from the results of Sec. III, i.e., $B^{(1)}=c_{6} / 2$ and $C^{(1)}=f_{4}$. The $C^{(i)}$ term is due to the rescaling. It is easy to see that $C^{(i)}=1-z$ by investigating the most singular term of Eq. (4.7), i.e., $C^{(i)}=f_{4}=1-z$. In the limit $x \rightarrow 0$, we find that Eq. (4.6) becomes

$$
\begin{aligned}
Q\left[\alpha, s_{i}, s_{1}\right] & =-\left[\frac{s_{1}^{\prime}(1)+g^{\prime \prime}(1) s_{1}(0)}{\alpha^{z-1}}\right] s_{i}(x)+\cdots \\
& =\frac{c_{6}}{2} s_{i}(x)+\cdots,
\end{aligned}
$$

where the relation

$$
c_{6}=-\frac{2\left[s_{1}^{\prime}(1)+g^{\prime \prime}(1) s_{1}(0)\right]}{\alpha^{z-1}}
$$

is given in Appendix C. This completes the proof for (4.9).

Using Eqs. (3.43) and (3.44), Eq. (4.5) becomes, for large $n$ and $p=m$,

$$
\begin{aligned}
u_{n+1} & =u_{n} \delta\left[1+\frac{\tau}{\kappa} v_{n}^{(1)}\right]+\cdots, \\
v_{n+1}^{(1)} & =v_{n}^{(1)}+v_{n}^{(2)}+\frac{v_{n}^{(1)}}{\kappa}+\cdots, \\
& \vdots \\
v_{n+1}^{(i)} & =v_{n}^{(i)}+v_{n}^{(i+1)}+\frac{v_{n}^{(1)} v_{n}^{(i)}}{\kappa}+\cdots, \\
\vdots & \\
v_{n+1}^{(m)} & =v_{n}^{(m)}+\frac{v_{n}^{(1)} v_{n}^{(m)}}{\kappa}+\cdots,
\end{aligned}
$$

where $i=1,2,3, \ldots, m$. The solution to this set of equations is straightforward; for $v_{n}^{(m)} \neq 0$, we have

$$
\begin{aligned}
& v_{n}^{(1)} \approx-\frac{m \kappa}{n}, \\
& v_{n}^{(2)} \approx-\frac{m(m-1) \kappa}{n^{2}}, \\
& v_{n}^{(i)} \approx-\frac{m ! \kappa}{(m-i) ! n^{i}}, \\
& u_{n+1} \approx u_{n} \delta\left[1-\frac{m \tau}{n}\right],
\end{aligned}
$$

which give

$$
\begin{aligned}
& \alpha_{n} \approx \alpha\left(1-\frac{m \kappa}{n}\right), \\
& \delta_{n} \approx \delta\left(1-\frac{m \tau}{n}\right) .
\end{aligned}
$$

This verifies the numerical results $(2.15)$ for $p=m$. Two comments are in order.

(1) One can easily verify that the terms neglected in Eqs. (4.5) and (4.11) are down by powers of $n$ and hence can be ignored.

(2) The result $[(4.11)-(4.13)]$ is independent of the choice of $s_{i}$ [i.e., $s_{i}(x)$ can be replaced by $s_{i}(x)+r_{i} s_{i-1}(x)$ for $i=2,3, \ldots]$.

\section{Functional relations}

In the derivation of the Jordan canonical form (3.25) we obtain

$$
s_{1}(x)=-\alpha_{z}\left(\frac{\partial \alpha_{z}}{\partial z}\right)^{-1} \frac{\partial g_{z}(x)}{\partial z},
$$

which implies that $\kappa$ and $\tau$ are connected to changes of $g_{z}(x), \alpha(z), \delta(z)$ in the $z$ direction. Here we shall elucidate this relationship.

Consider a function with extremum of the power $z-\epsilon$, 


$$
\begin{aligned}
f(x) & =|x|^{z-\epsilon} \\
& =|x|^{z} \exp \left(-\epsilon \frac{1}{2} \ln \left(x^{2}\right)\right) \\
& =|x|^{z} \sum_{k=0}^{\infty} \frac{\left(-\epsilon \frac{1}{2} \ln \left(x^{2}\right)\right)^{k}}{k !} .
\end{aligned}
$$

From (4.15) we see, for this type of function with large $n$ and small $\epsilon$, that

$$
\begin{aligned}
\alpha_{n} & \approx \alpha(z-\epsilon) \\
& \approx \alpha(z)\left(1-\frac{\epsilon}{\alpha(z)} \frac{\partial \alpha(z)}{\partial z}\right), \\
\delta_{n} & \approx \delta(z-\epsilon) \\
& \approx \delta(z)\left(1-\frac{\epsilon}{\delta(z)} \frac{\partial \delta(z)}{\partial z}\right),
\end{aligned}
$$

where only leading terms in $\epsilon$ are kept. These also indicate that for large $n, \alpha_{n}$ and $\delta_{n}$ are independent of $n$, as must be the case for the function (4.15). Now, let us consider (4.16), which is equivalent to (4.15). Using the Sterling formula for large $k$, we have

$$
f(x) \approx|x|^{z} \sum_{k}\left[\frac{-\epsilon \frac{1}{2} e \ln \left(x^{2}\right)}{k}\right]^{k} k^{-1 / 2},
$$

which is a good approximation only for the terms with large $k$. For a given large $n$ the relevant value of the $x$ variable is $x / \alpha^{n}$ where $|x| \approx[0,1]$. Therefore, the magnitude of an individual term in the sum in (4.19) is, for large $k$,

$$
\sigma(k) \approx\left(\frac{+\frac{1}{2} \epsilon e \ln \left(\alpha^{2 n}\right)}{k}\right)^{k} k^{-1 / 2} .
$$

The term that has the largest contribution to (4.19), for a given $n$, is given by

$$
\frac{\partial \sigma(k)}{\partial k}=0
$$

or

$$
k \approx n \epsilon \ln \alpha .
$$

For sufficiently small $\epsilon$, this term in fact dominates. For this value of $k$, we know, from (4.13),

$$
\begin{aligned}
\alpha_{n} & \approx \alpha(z)\left(1-\frac{k \kappa}{n}\right) \\
& \approx \alpha(z)(1-\epsilon \kappa \ln \alpha), \\
\delta_{n} & \approx \delta(z)\left(1-\frac{k \tau}{n}\right) \\
& \approx \delta(z)(1-\epsilon \tau \ln \alpha) .
\end{aligned}
$$

Comparing (4.17) with (4.22) and (4.18) with (4.23), we obtain

$$
\begin{aligned}
& \frac{\partial \alpha(z)}{\partial z}=\kappa(z) \alpha(z) \ln \alpha(z), \\
& \frac{\partial \delta(z)}{\partial z}=\tau(z) \delta(z) \ln \alpha(z),
\end{aligned}
$$

where the $z$ dependence in $\alpha(z), \delta(z), \kappa(z)$, and $\tau(z)$ are explicitly displayed. Of course, we can combine them to write

$$
\frac{\partial \alpha}{\partial \delta}=\frac{\kappa \alpha}{\tau \delta}
$$

\section{Numerical results}

Now we are ready to calculate $\kappa$ and $\tau$ using the renormalization-group analysis. To obtain the eigenvalues of the linear operator $L$, we consider the following truncation of our functional space (for $z=2, p=1$ case):

$$
\begin{aligned}
V(x)= & l_{1}+l_{2} x^{2}+l_{3} x^{2} \ln \left(x^{2}\right)+l_{4} x^{4}+l_{5} x^{4} \ln \left(x^{2}\right) \\
& +l_{6} x^{4}\left[\ln \left(x^{2}\right)\right]^{2}+l_{7} x^{6}+\cdots .
\end{aligned}
$$

Since we truncate the function space $F_{z=2}(p=1)$, we shall obtain two eigenvectors whose eigenvalues are very close to 1 , instead of two degenerate eigenvalues that form a Jordan canonical form of degree 2. The difference of the two eigenvectors is essentially $s(x)$. We can obtain $s(x)$ either by this method or, equivalently, by using Eq. (3.25). In Table $\mathrm{V}$ we give the result for the operators $h(x), t(x)$, $s(x)$, and their eigenvalues. Here 14 terms in (4.27) are used. It is easy to check numerically that some of the coefficients in Table $\mathbf{V}$ are very stable; in particular the coefficients of $x^{2}, x^{2} \ln \left(x^{2}\right)$ are very stable. The coefficient $l_{1}=1$ determines the normalization of $h(x)$ and $t(x)$. The choice of $l_{1}=0$ for $s(x)$ fixes the choice of $s(x)$.

For $z=2$ the coefficient $g_{1}$ in $g(x)$ is given by $g_{1}=-1.5276$. Taking $\beta_{1}=1.8728$ from Table $V$, we find, from Eq. (3.47), $\kappa=-0.4445$. In Table VI the various coefficients in Eqs. (3.33) and (3.34) are numerically determined using the eigenvectors obtained from Table $\mathrm{V}$. To see the accuracies of these coefficients, we check the theoretical relations (3.38), (3.45), and (3.46) with the numerical values in Table VI. This shows that the numbers in Table VI are typically reliable to 3 or 4 significant figures. Using (3.43) and (3.44) we obtain

$$
\kappa=-0.4445
$$

and

$$
\tau=0.3695 \text {. }
$$

Of course, better accuracy can be obtained if we include more terms in Eq. (4.27). $\kappa$ and $\tau$ are stable when we vary the functional space in a way such that terms in $F_{p}(p \geq 2)$ are excluded. However, some of the coefficients of the higher terms may become unstable. This implies that an optimum choice of the functional space may not have been achieved.

We can also obtain $\kappa$ and $\tau$ by using Eqs. (4.24) and (4.25). Comparing $z=2.0001$ and 2.0000 gives the same $\kappa$ and $\tau$. For $z=1.5, \quad \alpha=3.3887, \delta=3.8006$, $\kappa=-0.7622$, and $\tau=0.4206$. 
TABLE V. Eigenvalues and eigenvectors for the relevant operator $h(x)$ and the marginal operators $t(x)$ and $s_{1}(x)$ obtained from numerical solution to the Eqs. (3.13), (3.14), and (3.25).

\begin{tabular}{cccc}
\hline \hline Operator & $h(x)$ & $t(x)$ & $s_{1}(x)$ \\
Eigenvalue & 4.66920 & 1.00218 & 0.99780 \\
\hline 1 & 1.00000 & 1.0000 & 0.0 \\
$x^{2}$ & -0.32565 & 1.5274 & -0.68163 \\
$x^{2} \ln \left(x^{2}\right)$ & $1.3 \times 10^{-6}$ & $2.406 \times 10^{-5}$ & 1.8728 \\
$x^{4}$ & -0.03378 & -0.1965 & 0.16818 \\
$x^{4} \ln \left(x^{2}\right)$ & $-4.656 \times 10^{-2}$ & -0.03452 & -0.25455 \\
$x^{4}\left[\ln \left(x^{2}\right)\right]^{2}$ & $3.85 \times 10^{-4}$ & $3.1510 \times 10^{-3}$ & $-8.76 \times 10^{-4}$ \\
$x^{6}$ & 0.23156 & 0.39292 & 2.6078 \\
$\vdots$ & $\vdots$ & $\vdots$ & $\vdots$ \\
\hline \hline
\end{tabular}

\section{FURTHER GENERALIZATION}

\section{A. The $q \neq 0$ case}

To generalize the result of Secs. III and IV to the $p=$ arbitrary real number case is very difficult because the functional space for the operator $s_{p}(x)$ in general is not known. With some assumptions on the functional space and properties of $s_{p}(x)$, one can make an argument for the general $p$ case. However, in our viewpoint this is tantamount to assuming the answer. The numerical data for the small $p$ case is very good. So here we shall assume that (2.15) is true for small real number $p$. We then study some of the consequences of this assumption; numerical computation can then be used to check the consequences.

The idea is to repeat the method developed in Sec. IV C for logarithmic terms,

$$
\begin{aligned}
\Delta f & \approx|x|^{z}\left|\ln \left(x^{2}\right)\right|^{p+\epsilon} \\
& =|x|^{z}\left|\ln \left(x^{2}\right)\right|^{p} \sum_{k=0}^{\infty} \frac{\left[\epsilon \ln \left|\ln \left(x^{2}\right)\right|\right]^{k}}{k !} .
\end{aligned}
$$

For large $n$, the left-hand side gives the behavior

$$
\begin{aligned}
& \alpha_{n} \approx \alpha\left(1-\frac{\kappa(p+\epsilon)}{n}\right), \\
& \delta_{n} \approx \delta\left(1-\frac{\tau(p+\epsilon)}{n}\right) .
\end{aligned}
$$

Clearly, consistency requires the rhs to have the same behavior. Here we can use the same method as in Sec. IV C. For simplicity, let us consider the case where $p=0$. Then intuitively we expect, for $\Delta f \approx|x|^{z}\left[\ln \left|\ln \left(x^{2}\right)\right|\right]^{l}$,

$$
\lambda_{\infty}-\lambda_{n} \approx \delta^{-n}(\ln n)^{l \zeta}
$$

for large $n$, or

$$
\delta_{n} \approx \delta\left[1-\frac{l \zeta}{n \ln n}\right],
$$

where $\zeta$ is some constant, and similarly,

$$
\alpha_{n} \approx \alpha\left(1-\frac{l \gamma}{n \ln n}\right) .
$$

To obtain the large $n$ behavior for (5.1), we must determine the dominant $k$ term for a fixed, large $n$. It is straightforward to show that the dominant term has $k$ given by $k \approx \epsilon \ln \left[-\ln \left(x^{2}\right)\right]$. At the $n$th period doubling the relevant value of $x$ is $x \alpha^{-n}$ so that

$$
\begin{aligned}
k & \approx \epsilon \ln \left[2 n \ln \alpha-\ln \left(x^{2}\right)\right] \\
& \approx \epsilon\left[\ln n+\ln \ln \left(\alpha^{2}\right)\right] .
\end{aligned}
$$

This means

TABLE VI. Numerical values of the coefficients in the renormalization-group equation (3.35)-(3.37). They are expected to have an uncertainty of the order of $10^{-4}$. The coefficients are defined in Eqs. (3.33) and (3.34).

\begin{tabular}{lll}
\hline \hline$a_{1}=4.77165$ & $b_{1}=2.12359$ & $c_{1}=-1.45 \times 10^{-5}$ \\
$a_{2}=-6.76 \times 10^{-5}$ & $b_{2}=1.89698$ & $c_{2}=-3.567 \times 10^{-5}$ \\
$a_{3}=-2.97154$ & $b_{3}=3.51 \times 10^{-4}$ & $c_{3}=-9.444 \times 10^{-5}$ \\
$a_{4}=6.10694$ & $b_{4}=1.62486$ & $c_{4}=0.782225$ \\
$a_{5}=6.52128$ & $b_{5}=1.27808$ & $c_{5}=8.398 \times 10^{-5}$ \\
$a_{6}=-4.11755$ & $b_{6}=3.97157$ & $c_{6}=2.49958$ \\
& & \\
$d_{1}=0.404911$ & $e_{1}=-0.36059$ & $f_{1}=-4.89 \times 10^{-5}$ \\
$d_{2}=0.476548$ & $e_{2}=0.516992$ & $f_{2}=-7.50 \times 10^{-6}$ \\
$d_{3}=0.809838$ & $e_{3}=0.278433$ & $f_{3}=-1.20 \times 10^{-6}$ \\
$d_{4}=-1.55657$ & $e_{4}=1.29114$ & $f_{4}=-1.00003$ \\
\hline \hline
\end{tabular}




$$
\begin{aligned}
\delta_{n} & \approx \delta\left[-\frac{\epsilon \zeta\left[\ln n+\ln \ln \left(\alpha^{2}\right)\right]}{n \ln n}\right] \\
& \approx \delta\left[1-\frac{\epsilon \zeta}{n}\right)
\end{aligned}
$$

and similarly

$$
\alpha_{n} \approx \alpha\left(1-\frac{\epsilon \gamma}{n}\right) .
$$

Comparing with (5.2) and (5.3) for $p=0$, we obtain

$$
\zeta=\tau
$$

and

$$
\gamma=\kappa \text {. }
$$

This supports the formulas (1.2) and (1.3). We can check this by numerical iterations of some functions which have extrema of the form $\Delta f \approx|x|^{z}\left\{\ln \left[-a \ln \left(x^{2}\right)\right]\right\}^{q}$. From (5.7) and (5.8) we see that the approach is extremely slow,

$$
\tau \approx \zeta\left[1+\frac{\ln \left[a \ln \left(\alpha^{2}\right)\right]}{\ln n}\right) .
$$

If we consider the case in (5.7), i.e., $a=1$ and $z=2$, the correction term is quite substantial. Since the computational time increases like $2^{n}$, the maximum $n$ that is feasible via numerical iteration is $n \approx 20$. Then $\zeta$ will still be $20 \%$ away from $\tau$. In Figs. 6 and 7, the value of $\kappa$ and $\tau$ extracted from functions with $p=0$ and various values of $q$ are shown. The functions used have the form

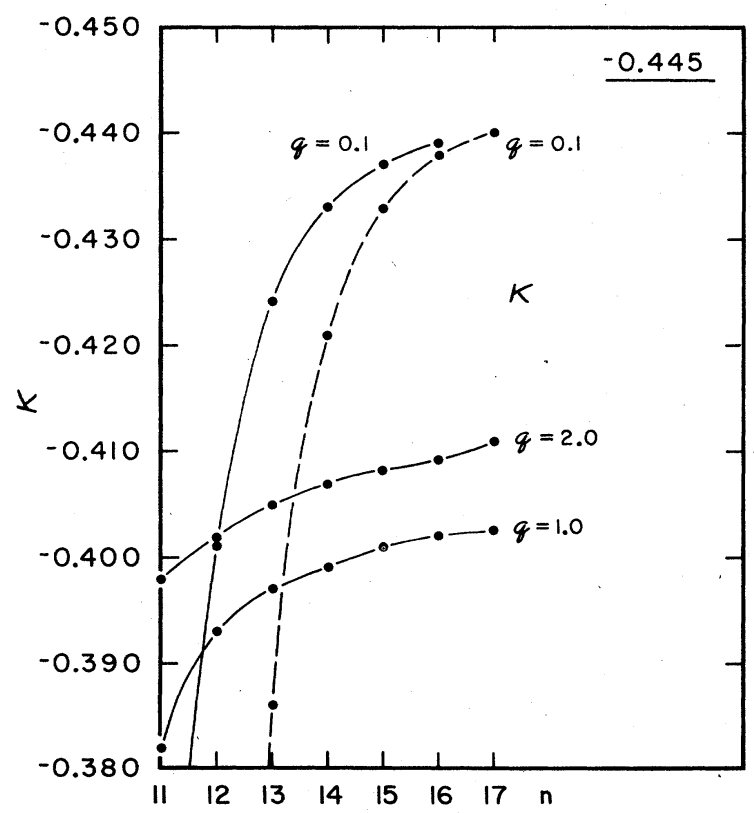

FIG. 6. Value of $\tau$ extracted from functions of the type (5.11) with different $q$ values where $p=0$. The curves are obtained from the following fits: (1) For the solid curves with $q=0.1$, 1.0, 2.0, the formula (5.12) is used; (2) For the dashed curve Eq. (5.13) is used. See the description in the text.

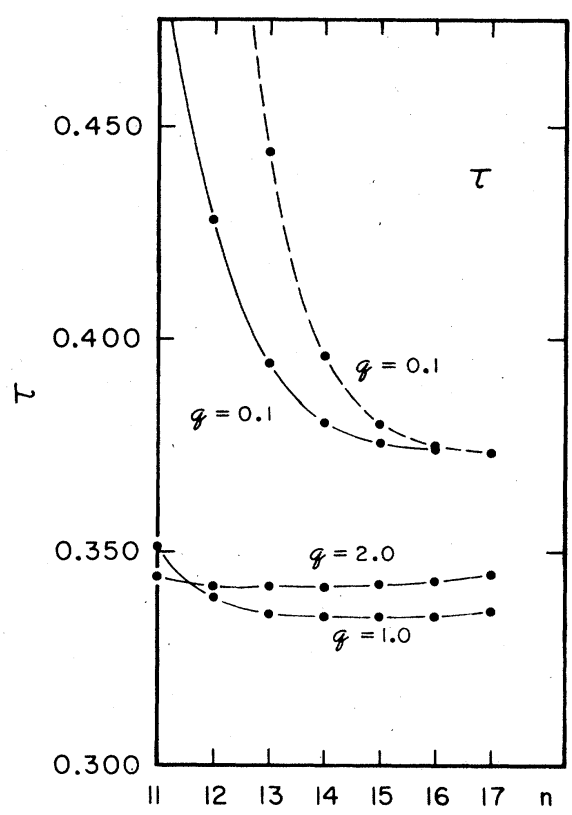

FIG. 7. Value of $\kappa$ extracted from functions of the type (5.11) with different $q$ values where $p=0$. The curves are obtained from fits that are described in the text.

$f(x) \approx \lambda\left\{1-(2 x-1)^{2}\left[c_{1}|\ln | \ln (2 x-1)^{2}||^{q}+c_{2}\right]\right\}$,

where $\left(q, c_{1}, c_{2}\right)$ takes the value $\left(2, \frac{1}{2}, 1\right),\left(1,1, \frac{1}{2}\right)$, and $\left(0.1, \frac{1}{2}, \frac{1}{2}\right)$. The $\tau$ value is extracted from the following fitting formula:

$$
\frac{\delta_{n}}{\delta}=1-\frac{\tau}{n} \frac{1}{\ln \left[n \ln \left(\alpha^{2}\right)\right]}\left(1+\frac{A}{n}+\frac{B}{n^{2}}\right),
$$

where the $\alpha_{n}$ data are generated numerically for $n=1,2, \ldots, 17$ (the solid curves in Fig. 6). We also consider the case where $a=+0.544 \ldots$ such that $\ln \left[a \ln \left(\alpha^{2}\right)\right] \approx 0$ so that the $(\ln n)^{-1}$ correction is suppressed. The dashed curve in Fig. 6 is for the function

$$
f(x)=\lambda\left\{1-(2 x-1)^{2} \ln \left[-a \ln (2 x-1)^{2}\right]^{0.1}\right\},
$$

where $q=0.1$ and $a=0.544 \ldots$ The $\tau$ value is extracted from the fitting formula:

$$
\frac{\delta_{n}}{\delta}=1-\frac{\tau}{n \ln n}\left(1+\frac{A^{\prime}}{n}+\frac{B^{\prime}}{n^{2}}\right) .
$$

Formulas similar to (5.12) and (5.13) are used to extract the $\kappa$ value in Fig. 7. We see that Figs. 6 and 7 do provide clean support for the asymptotic form (1.2) and (1.3). Of course, the accuracy is not as good as that for the $p \neq 0$, $q=0$ case, as expected from the slow approach to asymptotic behavior as $n$ becomes large. For functions whose extremum at $x=0$ has the form

$$
f(x) \approx|x|^{z}|\ln | \ln |\cdots| \ln x^{2}|\cdots|||^{r}
$$

the asymptotic behavior becomes exceedingly hard to 
reach. However, by repeating the above analytic argument, it clearly indicates that no new universal constants (besides $\alpha, \delta, \tau$, and $\kappa$ ) will emerge.

\section{B. The general symmetric case}

The above analysis allows us to extend our discussion to the general symmetric case. In fact, both the derivation and the result are very simple. Let us consider functions whose (symmetric) extremum at $x=0$ has the form of Eq. (1.8),

$$
f(x) \approx|x|^{z} b(x)
$$

If $b(x)$ modifies the form $|x|^{z}$ by more than a power, the universal period-doubling approach to chaos will be lost. If $b(x)$ modifies the form by a power, we can simply redefine the value of $z$. Hence, we need to consider only the case where, for any infinitesimal positive constant $v$, as $x \rightarrow 0$,

$$
\begin{aligned}
& |x|^{v} b(x) \rightarrow 0, \\
& |x|^{-v} b(x) \rightarrow \infty .
\end{aligned}
$$

At the $n$th bifurcation the relevant value of $x^{2}$ is given by $x_{n}^{2} \simeq \alpha^{-2 n}$. In general, the effective power of $|x|$ in the function $f(x)$ in Eq. (1.8) is given by

$$
\begin{aligned}
z(x) & =\frac{x}{f(x)} \frac{\partial f(x)}{\partial x} \\
& =z+\frac{\partial \ln b(x)}{\partial \ln |x|} .
\end{aligned}
$$

At the $n$th bifurcation, for asymptotic $n$,

$$
z(n)=z+\epsilon(n),
$$

where

$$
\epsilon(n)=\left.\frac{\partial \ln b(x)}{\partial \ln |x|}\right|_{\left|x_{n}\right| \approx \alpha^{-n}} .
$$

Now, the effective $\alpha$ and $\delta$ are the corresponding values at the effective power $z(n)$. Following Eqs. (4.17) and (4.18), we have, for asymptotically large $n$,

$$
\begin{aligned}
& \alpha_{n}=\alpha(z(n)) \simeq \alpha(z)\left(1+\frac{\epsilon(n)}{\alpha(z)} \frac{\delta \alpha(z)}{\delta z}\right), \\
& \delta_{n}=\delta(z(n)) \simeq \delta(z)\left(1+\frac{\epsilon(n)}{\delta(z)} \frac{\partial \delta(z)}{\partial z}\right),
\end{aligned}
$$

where terms of $\boldsymbol{O}\left(\epsilon^{2}\right)$ are neglected. Clearly, we expect, for any fixed $v$, as $x \rightarrow 0$ and $n \rightarrow \infty$,

$$
\frac{\epsilon(n)}{v} \rightarrow 0 \text {. }
$$

Using (1.4) and (1.5), we obtain the final result

$$
\begin{aligned}
& \alpha_{n} \simeq \alpha[1+\kappa \epsilon(n) \ln \alpha], \\
& \delta_{n} \simeq \delta[1+\tau \epsilon(n) \ln \alpha] .
\end{aligned}
$$

For example, if $b(x)$ has the following form: $b(x) \approx\left|\ln \left(x^{2}\right)\right|^{p}|\ln | \ln \left(x^{2}\right)||^{q}|\ln \ln | \ln \left(x^{2}\right)||^{r} \ldots$

then

$\epsilon(n)=\frac{-1}{\ln \alpha}\left[\frac{p}{n}+\frac{q}{n \ln (n)}+\frac{r}{n \ln (n) \ln \ln (n)}+\cdots\right)$.

In Eq. (5.14) we have consistently neglected terms of the order $\epsilon^{2}$ (or $n^{-2}$ ). To summarize, for functions whose extremum at $x=0$ has the form

$\Delta f_{\lambda} \approx|x|^{z}\left|\ln x^{2}\right|^{p}|\ln | \ln x^{2}||^{q}|\ln | \ln \left|\ln x^{2}\right|||^{r} \cdots$,

where $z, p, q, r \ldots$ are all real numbers and $z>1$, their behavior of period-doubling bifurcations is quantitatively universal as they approach the chaotic point, i.e., as $\lambda$ approaches the critical $\lambda_{c}$. At the $n$th bifurcation ( $n$ is asymptotically large) the value of $\lambda_{n}$ behaves as

$$
\lambda_{c}-\lambda_{n} \approx \delta^{-n} n^{\tau p}(\ln n)^{\tau q}(\ln \ln n)^{\tau r} \ldots
$$

and the typical distance away from the extremum at $\lambda=\lambda_{n}$ is

$$
d_{n} \approx \alpha^{-n} n^{\kappa p}(\ln n)^{\kappa q}(\ln \ln n)^{\kappa r} \ldots .
$$

It is amazing that no new universal constants are introduced when $q \neq 0, r \neq 0, \ldots$. The above derivation is very simple and general. The reader may ask, with such a derivation, why we need the renormalization-group analysis discussed earlier, since the $R G$ analysis is more complicated and less general. The reason is that the simple derivation given in this subsection is only a heuristic one. Specifically, we observe that there is more than one way to define the effective power $z(x)$, and hence $\epsilon(n)$. The $\epsilon(n)$ defined in Eq. (1.12) is justified only after the detailed RG analysis has been performed [e.g., the effective power $z(x)$ defined by $f(x) \approx|x|^{z(x)}$ is incorrect]. The renormalization-group analysis plus the above derivation give us the universal quantitative properties of the general symmetric case.

\section{The asymmetric case}

For the sake of completeness, let us consider other type of functions that can exhibit cascades of period doubling, namely, functions that have asymmetric extrema, as those given in (1.13). Here we shall briefly summarize the universal metric properties of such functions. ${ }^{4}$ It is clear that there are two universal functions $g_{1}(x)$ and $g_{2}(x)$ such that

$$
\begin{aligned}
& \alpha_{1} g_{1}\left(g_{1}\left(x / \alpha_{1}\right)\right)=g_{2}(x), \\
& \alpha_{2} g_{2}\left(g_{2}\left(x / \alpha_{2}\right)\right)=g_{1}(x) .
\end{aligned}
$$

We can introduce $\sigma=\alpha_{1} \alpha_{2}$ and combine them into one equation for the universal function $\bar{g}(x)$,

$$
\sigma \bar{g}(\bar{g}(\bar{g}(\bar{g}(x / \sigma))))=\bar{g}(x),
$$

where $\bar{g}(x)$ and $\sigma$ are both functions of $z$ and $\epsilon$, 
$=\widetilde{s}(x)+r t(x)$ for some constant $r$. Then

$$
Q[\alpha, h, \widetilde{s}]=\widetilde{a}_{4} h(x)+\widetilde{b}_{4} t(x)+\widetilde{c}_{4} \widetilde{s}(x)+\cdots
$$

and we want to find the relationship of the old set of coefficients to the new set of coefficients. Recall

$$
Q[\alpha, h, t]=a_{2} h(x)+b_{2} t(x)+c_{2} s(x)+\cdots,
$$

where $c_{2}=0$ since $Q[\alpha, h, t]$ has no logarithmic terms. Since the operator $Q[\alpha, h, s]$ is linear in $s(x)$,

$$
\begin{aligned}
Q[\alpha, h, s]= & Q[\alpha, h, \widetilde{s}]+r Q[\alpha, h, t] \\
= & \left(a_{4}+r a_{2}\right) h(x)+\left(\widetilde{b}_{4}+r b_{2}-r \widetilde{c}_{4}\right) t(x) \\
& +\widetilde{c_{4}} \widetilde{s}(x)+\cdots .
\end{aligned}
$$

Comparing (A1) and (A4) we find

$$
\begin{aligned}
& a_{4}=\widetilde{a}_{4}+r a_{2}, \\
& b_{4}=\widetilde{b}_{4}+r b_{2}-r \widetilde{c}_{4} .
\end{aligned}
$$

It is straightforward to generalize this argument to obtain the following results. $a_{1}, b_{1}, a_{2}, b_{2}, a_{3}, b_{3}, c_{4}$, and $c_{5}$ as well as $d_{1}, d_{2}, d_{3}, e_{1}, e_{2}, e_{3}, f_{1}, f_{2}, f_{3}$, and $f_{4}$ are invariant under the change of $s(x)$, while

$$
\begin{aligned}
& \widetilde{a}_{4}=a_{4}-r a_{2}, \\
& \widetilde{a}_{5}=a_{5}-r a_{3}, \\
& \widetilde{a}_{6}=a_{6}-2 r a_{5}+r^{2} a_{3}, \\
& \widetilde{b}_{4}=b_{4}-r b_{2}+r c_{4}, \\
& \widetilde{b}_{5}=b_{5}-r b_{3}+r c_{5}, \\
& \widetilde{b}_{6}=b_{6}-2 r b_{5}-2 r^{2} c_{5}+r^{2} b_{3}+r c_{6}, \\
& \widetilde{c}_{6}=c_{6}-2 r c_{5}, \\
& \widetilde{d}_{4}=d_{4}-r d_{3}, \\
& \widetilde{e}_{4}=e_{4}-r e_{3}+r f_{4} .
\end{aligned}
$$

\section{APPENDIX B}

The operator $I\left[\alpha_{n}, g_{n}\right]$ in Eq. (3.27) can be written as a product of two operators,

$$
I\left[\alpha_{n}, g_{n}\right]=R\left[\epsilon_{n}, I\left[\alpha, g_{n}\right]\right],
$$

where

$$
R[\epsilon, f(x)]=(1+\epsilon) f\left(\frac{x}{1+\epsilon}\right)
$$

and

$$
I[\alpha, f(x)]=-\alpha f(f(-x / \alpha)) .
$$

Note that

$$
R\left[\epsilon, f_{1}+f_{2}\right]=R\left[\epsilon, f_{1}\right]+R\left[\epsilon, f_{2}\right] .
$$

Also $R$ and $I$ commute, i.e.,

$$
\begin{aligned}
I[\alpha, R[\epsilon, f]] & =I\left[\alpha,(1+\epsilon) f\left[\frac{x}{1+\epsilon}\right]\right] \\
& =-\alpha(1+\epsilon) f\left[f\left(\frac{-x}{(1+\epsilon) \alpha}\right)\right] \\
& =R\left[\epsilon,-\alpha f\left[f\left(\frac{-x}{\alpha}\right)\right]\right] \\
& =R[\epsilon, I[\alpha, f]] .
\end{aligned}
$$

In Sec. III Eqs. (3.35)-(3.37) are obtained by evaluating $I\left[\alpha, R\left[\epsilon_{n}, g_{n}\right]\right]$. We can obtain the same equations by evaluating $R\left[\epsilon_{n}, I\left[\alpha, g_{n}\right]\right]$. Comparing the two sets of equations and equating the coefficients of the terms to quadratic powers, i.e., to $u_{n}^{2}, u_{n} v_{n}, u_{n} w_{n}, v_{n} w_{n}, v_{n}^{2}$, $w_{n}^{2} \epsilon_{n} u_{n}, \epsilon_{n} v_{n}, \epsilon_{n} w_{n}$, we obtain the relations (3.45). The calculation is straightforward. In particular, it is easy to see that the coefficients $a_{2}=b_{3}=c_{5}=0$ since the corresponding terms are absent in $R\left[\epsilon_{n}, I\left[\alpha, g_{n}\right]\right]$.

\section{APPENDIX C}

Let us differentiate (3.25) with respect to $x$, divide the resulting equation by $|x|^{z-1}$, and then take the $x \rightarrow 0$ limit. This straightforward procedure gives the following relation:

$s^{\prime}(1)+g^{\prime \prime}(1) s(0)=\left[(z-1)-\frac{2 \beta_{1} \ln \alpha}{g_{1}}\right] \alpha^{z-1}$,

where $g_{1}$ is the coefficient of $|x|^{z}$ in $g(x)$ as given in (3.8) and $\beta_{1}$ is the coefficient of $|x|^{2} \ln \left(x^{2}\right)$ in $s(x)$,

$$
s(x)=\beta_{1}|x|^{z} \ln \left(x^{2}\right)+\cdots \cdot
$$

Next, let us turn our attention to $Q[\alpha, s, s]$,

$$
Q[\alpha, s, s]=c_{6} s(x)+\cdots .
$$

Equating the coefficient of the $|x|^{z} \ln \left(x^{2}\right)$ term, we obtain

$$
\frac{-2\left[s^{\prime}(1)+g^{\prime \prime}(1) s(0)\right]}{\alpha^{2-1}}=c_{6} .
$$

We observe that the above procedure implicitly assumes that all irrelevant operators do not contain the term $|x|^{z} \ln \left(x^{2}\right)$. This is because any eigenoperator that contains the $|x|^{z} \ln \left(x^{2}\right)$ term should have eigenvalue one (see Sec. IV B). Therefore

$$
\kappa^{-1}=-\left(\frac{c_{6}}{2}+z-1\right)=\frac{-2 \beta_{1} \ln \alpha}{g_{1}} .
$$

Recall that we can choose $r$ such that

$$
\begin{aligned}
s(x) & =-\alpha_{z}\left(\frac{\delta \alpha_{z}}{\delta z}\right)^{-1} \frac{\delta g_{z}}{\delta z}(x) \\
& =-\alpha_{z}\left(\frac{\delta \alpha_{z}}{\delta z}\right)^{-1}\left[\frac{g_{1}}{2}|x|^{z} \ln \left(x^{2}\right)+\cdots\right),
\end{aligned}
$$




$$
\bar{g}(x)= \begin{cases}1+(1+\epsilon) g_{1}|x|^{z}+\cdots, & x>0 \\ 1+(1-\epsilon) g_{1}|x|^{z}+\cdots, & x<0 .\end{cases}
$$

Of course, for $\epsilon=0, \bar{g}(x)=g_{z}(x)$ and $\sigma(z, \epsilon=0)=\alpha^{2}(z)$. For $\epsilon \neq 0$, besides $\bar{g}(z, \epsilon, x)$ and $\sigma(z, \epsilon)$, there is a corresponding set of eigenvectors and eigenvalues, which can be obtained from

$$
\begin{aligned}
& \sigma\left\{\bar{g}^{\prime}(\bar{g}(\bar{g}(\bar{g}(x / \sigma))))\right. \\
& \times\left[\overline { g } ^ { \prime } ( \overline { g } ( \overline { g } ( x / \sigma ) ) ) \left[\bar{g}^{\prime}(\bar{g}(x / \sigma)) \bar{h}(x / \sigma)\right.\right. \\
& +\bar{h}(\bar{g}(x / \sigma))] \\
& \quad+\bar{h}(\bar{g}(\bar{g}(x / \sigma)))] \\
& +\bar{h}(\bar{g}(\bar{g}(\bar{g}(x / \sigma))))\}=\lambda \bar{h}(x) .
\end{aligned}
$$

For each value of $\epsilon$, there are two solutions to Eq. (5.18): $g_{1}(x)$ and $g_{2}(x)$. For each $g_{i}(x), i=1,2$, there is a set of eigenvectors $h_{i, j}$ with corresponding eigenvalues $\lambda_{j}$, $i=1,2$ and $j=1,2,3, \ldots$ For $\epsilon=0$ the leading operators coincide with $h(x), \bar{h}_{11}=\bar{h}_{21}=h(x)$, and the corresponding eigenvalue $\lambda(z, \epsilon=0)=\delta^{2}$. In fact, it turns out that the universal functions $g_{i}(x)$ and the eigenoperators are both functions of the rescaled $x$ only; to explain this, let us consider $z=2$, then

$$
g_{1}(x)= \begin{cases}1+\sum_{i} g_{1}^{(i)}\left[(1+\epsilon) x^{2}\right]^{i}, & x>0 \\ 1+\sum_{i} g_{1}^{(i)}\left[(1-\epsilon) x^{2}\right]^{i}, & x<0\end{cases}
$$

where the coefficients $g_{1}^{(i)}$ are the same for $x \gtrless 0 . g_{2}(x)$ and $h_{i j}(x)$ have similar behaviors. As an example, for $\epsilon=0.1$, we have

$$
\begin{aligned}
& \sigma(z=2, \epsilon=0.1)=(2.5048984)^{2}, \\
& \lambda(z=2, \epsilon=0.1)=(4.6773189)^{2} .
\end{aligned}
$$

We leave it as an exercise for the reader to consider the case where the extremum has both the logarithms and the asymmetry.

\section{REMARKS AND CONCLUSIONS}

One-dimensional maps that exhibit repeated perioddoubling bifurcations are being analyzed in some detail here. It is shown that the universal metric properties of this period-doubling behavior are in general determined by four universal constants, $\alpha, \delta, \kappa$, and $\tau$; the general symmetric case is obtained by a simple derivation (Sec. VB). The justification of this intuitively clear derivation comes from the more careful and detailed analysis in Secs. III and IV; here the RG method plays a crucial role. The RG method is useful in two ways. It allows us to analytically derive two functional relations among $\alpha, \delta, \kappa$, and $\tau$. This provides a valuable insight into the underlying structure of the universality of 1D maps. The RG method also makes the numerical determination of the universal constants much easier. To determine $\kappa, \tau$ numerically without the use of RG, we simply pick a function and start to iterate it. The computational time goes like $2^{n}$ as the accuracy of $\kappa$ and $\tau$ improve like $n^{-c}$ for some constant $c$ (in contrast, the accuracy of $\alpha$ and $\delta$ improve like $a^{-n}$ for some constant $a$ ). If we use the RG method the computational time goes like $n^{2}$, which is clearly much more efficient. For example, if we want $\kappa$ and $\tau$ to be determined to four significant figures, the RG method requires only a few seconds of central-processing unit time on the IBM 3081 while the brute-force approach requires approximately an hour for each function to be iterated.

The results of the above analysis are also applicable to the study of band bifurcation on the chaotic side, i.e., $\lambda>\lambda_{c}$. This is a reflection of the simplicity of 1D maps in comparison to higher-dimensional maps. It will be interesting to consider the effect of noise on systems with logarithmic modifications. We observe that all functions considered in our analysis are at least once differentiable.

The RG analysis hinges on one important feature. The $g(x)$ function and hence the operator $L$ belong to the "power-of- $x$ " space. We find that the space in which solutions to $L$ exist is in general larger than the space in which $L$ belongs, i.e., solutions to the equation with the operator $L$ can contain functional forms that are absent in the power-of- $x$ space. Such a feature is quite common in physics. For example, consider Newton's equation for the earth moving around the sun. The differential operator is spherically symmetric and circles are clearly solutions of the equation, but so are elliptical orbits. In quantum-field theory there are ample examples where the solution of an equation spontaneously breaks the symmetry of the equation.

Our analysis clearly raised the question concerning functional space. In fact, this issue is already present in the Feigenbaum analysis. For a general $z$ the functional space for $g_{z}(x)$ is not known. In general, a necessary condition on the functional space $F$ is that, given a function $g_{z}(x)$ defined in $F$, the operation on the function $g_{z}(x)$ does not take it out of $F$. However, $g_{z}(x)$ of the form

$$
g_{z}(x)=1+g_{1}|x|^{2}+g_{2}|x|^{2 z}+g_{3}|x|^{3 z}+\cdots
$$

(for $z \neq 2$ ) is in general inappropriate in the determination of $\alpha(z)$. This issue of functional space clearly requires further investigation. A better understanding of the space $F_{p}$ and its generalization $F_{p, q, \ldots}$ will be useful.

\section{ACKNOWLEDGMENTS}

We thank Mitchell Feigenbaum, Alex Hansen, Toichiro Kinoshita, and Kenneth Wilson for useful discussions. This research is supported by National Science Foundation Grants Nos. PHY-82-09011 and PHY-80-22200.

\section{APPENDIX A}

If $s(x)$ is a solution of the equation

$$
L[\alpha, s]=s(x)+t(x),
$$

where $t(x)$ is given by Eq. (3.14), so is any linear combination of $s(x)$ and $t(x)$. Let us consider the equation

$$
\begin{aligned}
Q[\alpha, h, s] & =Q[\alpha, s, h] \\
& =a_{4} h(x)+b_{4} t(x)+c_{4} s(x)+\cdots .
\end{aligned}
$$

Suppose we change to another basis $s(x)$, where $s(x)$ 
where Eq. (3.8) is used. Combining (C2), (C4), and (C5), we obtain

$$
\beta_{1}=-\alpha_{z}\left(\frac{\delta \dot{\alpha}_{z}}{\delta z}\right)^{-1} \frac{g_{1}}{2}
$$

or

$$
\frac{1}{\alpha_{z}} \frac{\delta \alpha_{z}}{\delta z}=\kappa \ln \alpha_{z},
$$

${ }^{1}$ See, e.g., P. Collet and J.-P. Eckmann, Iterated Maps of the Interval as Dynamical Systems (Birkhauser, Cambridge, Mass., 1980); J. P. Crutchfield, J. D. Farmer, and B. A. Huberman, Phys. Rep. 92, 45 (1982).

${ }^{2}$ M. Feigenbaum, J. Stat. Phys. 19, 25 (1978); 21, 669 (1979).

${ }^{3}$ E. N. Lorenz, Ann. N. Y. Acad. Sci. 357, 282 (1980); J. Crutchfield, D. Farmer, R. Shaw, G. Jones, and R. Donnelly, Phys. Lett. 76A, 1 (1980).

${ }^{4}$ H. Kawai, T. Kinoshita, and S.-H. H. Tye (unpublished); M. Feigenbaum (private communication).
${ }^{5}$ Our analysis follows closely the formalism developed in K. G. Wilson, Rev. Mod. Phys. 47, 773 (1975), where earlier reference to the subject can also be found.

6S. Coleman and E. Weinberg, Phys. Rev. D 7, 1888 (1973).

${ }^{7}$ E. N. Lorenz, J. Atmos. Sci. 20, 130 (1963).

${ }^{8}$ M. J. Feigenbaum, Phys. Lett. 74A, 375 (1979); Commun. Math. Phys. 77, 65 (1980); P. Collet, J.-P. Eckmann, and L. Thomas, ibid. (to be published); M. Nauenberg and J. Rudnick (unpublished).

${ }^{9}$ See, e.g., M. J. Fiegenbaum, Physica (Utrecht) 7D, 16 (1983). 